\title{
Horst W. Schmollinger/Richard Stöss Bundestagswahlen und soziale Basis politischer Parteien in der Bundesrepublik III: Das Scheitern der sozialliberalen Koalition
}

\section{Vorbemerkung}

\section{1 Ökonomie und Politik im Kapitalismus}

Die ersten beiden Teile dieses Artikels erschienen in PROKLA25 und 26(1976/77) anläßlich der Bundestagswahl 1976. Das war vor acht Jahren. Die Begeisterung für die marxistische politische Ökonomie hatte sichtlich nachgelassen, und die intellektuelle marxistische Linke schlitterte gerade in ihre Identitätskrise ("Krise des Marxismus«). Vorangegangen war eine jahrelange Staatsdiskussion, die sich auf hohem Niveau und fernab jedes konkreten Praxisbezugs damit beschäftigte, die Rolle und die Notwendigkeit des bürgerlichen Staates begrifflich schlüssig aus der kapitalistischen Produktionsweise zu erklären (abzuleiten). Dem lag die Überzeugung zugrunde, daß in kapitalistischen Gesellschaften alle ökonomisch-sozialen, politisch-rechtlichen und ideologisch-kulturellen Erscheinungen einen einheitlichen, auf die Reproduktion des Kapitalismus funktional bezogenen Wirkungszusammenhang darstellten, und $\mathrm{da} ß$ sich folglich ökonomische Krisen auf die gesellschaftliche Ordnung insgesamt auswirkten. So war es selbstverständlich, die Existenzbedingungen der politischen Institutionen und Vorgänge aus der kapitalistischen Ökonomie heraus zu begründen. Dies galt auch für die politischen Parteien.

Das Interesse der »Neuen Linken « an politischen Parteien war außerordentlich gering, in wissenschaftlicher wie in politischer Hinsicht. Schon 1967 hatte Johannes Agnoli die Parteien in seiner »Transformation der Demokratie« als integrale Bestandteile des politischen Herrschaftsprozesses im Kapitalismus dargestellt, und die außerparlamentarische Opposition der sechziger Jahre hielt es weithin mit der Devise von Otto Rühle: „Die Revolution ist keine Parteisache!«. So oder so: Parteien und Parlamente galten als Ausdruck und Mittel bürgerlicher Herrschaft. Als solche wurden sie erstmalig umfassend von Michael Th. Greven in der 1977 erschienenen Studie »Parteien und politische Herrschaft« analysiert. Greven zeigte vor allem die Funktionalität der Organisationsform Partei für die politische Herrschaft im Kapitalismus auf und zog daraus den Schluß, daß eine Gefährdung der kapitalistischen Ordnung von den Parteien nicht zu erwarten sei, eher dagegen von den »authentischen antikapitalistischen Sozialbewegungen «.

Das Bild vom hyperstabilen und hinsichtlich seiner Integrationsfähigkeit nahezu omnipotenten bundesdeutschen Kapitalismus hatte eine gewisse empirische Evidenz. So waren die Voraussagen marxist ischer Ökonomen, daß mit der wachsenden Krisenhaftigkeit des Kapitalismus auch schärfere Formen des Klassenkampfes »auf der Tagesordnung « stünden, nicht eingetreten. Sozialdemokratie und Gewerkschaften wurden bereits in der Krise 1966/67 als »Arzt am Krankenbett des Kapitalismus« identifiziert, und an dieser Einschätzung sollte sich auch während der sozialliberalen Koalition wenig ändern. Im Gegenteil: Es war damals gerade die SPD, die die politische und juristische Repression der Linken einleitete. Die Union segelte Mitte der siebziger Jahre im Aufwind der Wählergunst, bei der Bundestagswahl 1976 
wurde sie wieder stärkste Partei im Bundestag, und die bundesrepublikanische Linke dümpelte konzeptions- und resonanzlos vor sich hin.

So beschrieb Wolf-Dieter Narr im Wahljahr 1976 die Bundesrepublik im Editorial zu dem von ihm 1977 herausgegebenen Sammelband »Auf dem Weg zum Einparteienstaat« als »geradezu ermüdend normal und stabil«. Er sprach damals vom»musterhaften Parteienstaat«, von der »etatistischen großen Koalition« der Bundestagsparteien und bewertete die Bundesrepublik als die »Stabilitäts- und Ordnungszelle Europas«. Auch Narr sah die politischen Parteien als Herrschaftsagenturen des bestehenden Systems (»Systemparteien«, »Staatsparteien«, "Einheitspartei fdGO «) und glaubte, daß andere (als die herrschenden) Parteien unter den gegebenen Bedingungen nicht den Hauch einer Chance hätten. Dies sollte sich drei Jahre später ändern.

Aus heutiger Sicht muß das Theorem vom systemfunktionalen Wirkungszusammenhang von Ökonomie, Politik und Ideologie im Kapitalismus mit Blick auf seine wissenschaftlichen und politischen Folgewirkungen als verhängnisvolle Sackgasse beurteilt werden.

Politisch mag es zur Rationalisierung der eigenen Hilflosigkeit beigetragen haben, es bildete jedoch auch eine Barriere für die Entwicklung von politischen Alternativen. Denn wenn Politik zwangsläufig der Reproduktion der bestehenden Produktionsverhältnisse dient, wenn Politik nur eine abhängige Variable von ÖKonomie darstellt, dann muß jede Aktivität innerhalb der bestehenden politischen Ordnung als aussichtslos erscheinen, dann bleibt letztlïch nur der Rekurs auf die russische oder die chinesische Revolution oder auf den Guerilla-Krieg. Das Scheitern der revolutionären maoistischen Kaderparteien und der RAF bzw. der Revolutionären Zellen und der unerwartete kometenhafte Aufstieg der sogenannten »Neuen Sozialen Bewegungen «, der Grünen und Alternativen in der zweiten Hälfte der siebziger Jahre bewies, daß sich die Theorie allzuweit von der Realität entfernt hatte.

In wissenschaftlicher Hinsicht krankte das Theorem vom systemfunktionalen Wirkungszusammenhang von Ökonomie, Politik und Ideologie vor allem daran, daß die widersprüchliche Einheit (Dialektik) von Ökonomie und Politik (vgl. dazu Blanke, S. 136 ff.) nicht - oder wenigstens doch zu spät - erkannt und daher das wichtige Problem der Vermittling von Ökonomie und Politik fast vollkommen ausgeblendet worden war. Ökonomie und Politik folgen in bürgerlich-kapitalistischen Gesellschaften nämlich widersprüchlichen Herrschafts- und Strukturprinzipien: In der Ökonomie gilt das Privateigentum an Produktionsmitteln, herrscht das Kapital über die Lohnarbeit. In der Politik dagegen gilt die formelle rechtliche und politische Gleichheit. Die Ausübung der staatlichen Macht ist nicht einer Klasse vorbehalten, die bürgerlichen Verfassungen sehen vielmehr vor, daß die Machthaber von den freien und gleichen Staatsbürgern periodisch gewählt bzw. bestätigt werden. In dieser Widersprüchlichkeit beziehen sich Ökonomie und Politik jedoch aufeinander. Der durch ökonomische Gesetzmäßigkeiten (Wertbewegung) gesteuerte Prozeß der Produktion und Verteilung bedarf einer außerökonomischen, eben politischen Instanz, die als Garant allgemeiner Produktionsvoraussetzungen Defizite des durch den Markt naturwüchsig geregelten Produktionsund Verteilungsprozesses auf dem Wege der Umverteilung von Geld behebt (Sozialpolitik, Strukturpolitik, Umweltpolitik, Bildungspolitik usw.) und die als allgemeine Zwangsgewalt über die Einhaltung der Verkehrsregeln auf dem Wege der Rechtssetzung und Rechtssicherung wacht (Blanke/Jürgens/Kastendiek, S. 414 ff.).

Die Funktion der Politik besteht also darin, daß sie Leistungen erbringt, die die Ökonomie von sich aus nicht erbringen kann, derer sie aber bedarf. Daraus wurde (und wird) der voreilige Schluß gezogen, daß sich Politik notwendigerweise immer funktional auf die Ökonomie 
bezieht, und übersehen, daß es sich hierbei nur um einen funktionalen Imperativ in Bezug auf das Ergebnis von Politik handeln kann. Da die Verkehrsformen in der politischen Sphäre gerade nicht unmittelbar der Kapitallogik unterliegen, besteht die Möglicbkeit, daß das Ergebnis der Politik diesem funktionalen Imperativ nur bedingt entspricht oder sogar widerspricht. Der politische Prozeß bietet keineswegs automatisch die Gewähr dafür, daß sich seine Steuerungs-, Anpassungs- und Modernisierungsleistungen sowie die Resultate der Rechtssicherung auch tatsächlich als gesellschaftlich nützlich im Sinne der kapitalistischen Reproduktion erweisen. Zwar signalisieren gesamt wirtschaftliche Indikatoren (Arbeitslosigkeit, Produktivität etc.) den Erfolg staatlicher Politiken. Über deren Nützlichkeit entscheiden jedoch unmittelbar die Staatsbürger, und zwar auch nach höchst subjektiven Kriterien(z.B. persönliche Zufriedenheit, Zukunftserwartungen, Einkommen, Lebensstandard).

Die Vermittlung von Ökonomie und Politik stellt mithin einen sehr differenzierten und widersprüchlichen, auf Anpassung zielenden Prozeß dar, dessen Ergebnis jedoch keineswegs von Anfang an feststeht. Wegen der prinzipiellen Bindung der Politik an die kapitalistische Ökonomie ist die Durchsetzung von nicht kapitalkonformen Interessen zwar außerordentlich erschwert, sie ist jedoch nicht grundsätzlich ausgeschlossen (zu den Handlungsgrenzen des Staates: Blanke/Jürgens/Kasiendiek, S. 436 ff.).

\subsection{Zur Rolle der Parteien im Prozeß der Vermittlung von Ökonomie und Politik}

Voraussetzung für die effiziente Vermittlung von Ökonomie und Politik ist ein auf die(staatliche) Zentralgewalt orientiertes System von Institutionen und Strukturen, dessen Aufgabe darin besteht, die Rechtsordnung zu gestalten und politische Konzepte zu formulieren und durchzuführen. Die letztverbindlich entscheidende Instanz, die auch über die Einhaltung der Beschlïsse wacht und gegebenenfalls Sanktionen verhängt, ist der Staat.

Da das Wesen der Politik darin besteht, Recht zu setzen und Defizite der durch ökonomische Gesetzmäßigkeiten geregelten kapitalistischen Produktion und Verteilung zu korrigieren, wird der politische Prozeß einerseits von subjektiven Bedürfnissen und Interessen hinsichtlich der Gestaltung der Rechtsordnung und der allgemeinen Produktionsvoraussetzungen und andererseits von den objektiven ökonomischen Rahmen bedingungen (finanzieller Umverteilungsspielraum, Krisenprophylaxe) geprägt. Das für die Vermittlung von Ökonomie und Politik notwendige System von Institutionen und Strukturen muß die Artikulation, Aggregation und Vermittlung von sozio-ökonomischen Interessen und gesellschaftsgestaltenden Konzeptionen gewährleisten, und der Staat steht vor der doppelten Aufgabe, gesellschaftlich vorhandene Bedürfnisse weitestgehend zu befriedigen und für die möglichst umfassende Akzeptanz seiner Tätigkeit zu sorgen (siehe dazu auch Stöss, S. 148 ff.).

Hier setzt die allgemeine Begriffsbestimmung von politischen Parteien an: Parteien sind Organisationen, in denen sich Menschen mit gleichartigen bzw. übereinstimmenden ökonomisch-sozialen Interessen und/oder politischen Zielen zusammenfinden, um diese Interessen und Ziele in politische Praxis umzusetzen. Dazu bedarf es in der Regel der Macht oder eines Machtanteils im Staat. Parteien streben folglich zumeist danach, die Ämter im Staat mit ihren Anhängern zu besetzen. Tragen sie tatsächlich staatliche Verantwortung, dann sind sie gezwungen, das Ergebnis staatlicher Tätigkeit gegenüber ihren (potentiellen) Anhängern zu rechtfertigen. Nun gibt es verschiedene(Typen von) Parteien, die sich hinsichtlich der Sozialstruktur ihrer Anhänger (Wähler, Mitglieder), der Organisationsform und der Żiele unterscheiden. Parteien können ihre Interessen und Ziele innerhalb der bestehenden politischen 
und ökonomischen Ordnung durchsetzen, sie können aber ebensogut den Versuch unternehmen, die bürgerlich-kapitalistische Ordnung sowohl in der ökonomischen als auch in der politischen Sphäre mit dem Ziel zu bekämpfen, eine andere Ordnung zu errichten.

Die Behauptungen, politische Parteien seien integraler Bestandteil der politischen Herrschaft im Kapitalismus und dienten nur der Aufrechterhaltung der bestehenden Produktionsweise, sind daher begrifflich falsch und durch die historische Realität auch nicht gedeckt. Sogibt es (unbeschadet möglicher Verbote) in allen bürgerlich-kapitalistischen Gesellschaften antikapitalistische Parteien, die ihrer Zielsetzung nach auf eine sozialistische Gesellschaft hinarbeiten (was nun wiederum nicht heißen soll, daß die Revolution eine Parteisache ist und daß gesellschaftliche Veränderungen nicht auch und vielleicht sogar erfolgreicher von sozialen Bewegungen ausgehen können). Dies galt und gilt auch für das (west-) deutsche Parteiensystem. Der Begriff Partei ist qualitativer Natur, quantitative Merlsmale, wie beispielsweise Größe oder Einfluß, spielen bei der Begriffsbildung keine Rolle. Vielmehr besteht gerade darin eire der wichtigsten Aufgaben historisch-soziologischer Parteienforschung, zu ermitteln, unter welchen ökonomischen und politischen Rahmenbedingungen sich welche Parteien bzw. Parteitypen durchsetzen. In bewußter Abgrenzung gegen die kapitalfunktionale Bestimmung der Partei als Ausdruck und Mittel bürgerlicher Herrschaft verwenden wir folgende Definition:

»Politische Parteien sind organisatorische Zusammenschlüsse von sozial und/oder interessenmäßig und/oder durch gemeinsame politische Ziele verbundenen Teilen des Volkes (auf der Grundlage eines Programms) und streben danach, die Ausübung von staatlicher Macht bzw. ökonomischer und außerökonomischer Herrschaft in ihrem Sinne zu gestalten.«(Stöss, S. 41 ff.)

\section{Struktâurprobleme des (west-) deutschen Parteiensystems}

\subsection{Konsens und Konflikt}

Die Gesellschaft der Bundesrepublik ist eine Klassengesellschaft ohne offenen Klassenkampf. Dieser Umstand ist in logischer wie in historischer Hinsicht erklärungsbedürftig: Wie ist es möglich, daß der antagonistische Widerspruch zwischen Lohnarbeit und Kapital nicht zum politischen Kampf der Ausgebeuteten gegen die Ausbeuter, der proletarischen Partei gegen die Partei der Bourgeoisie führt? Wie löst die bürgerlich-kapitalistische Gesellschaft den Widerspruch zwischen sozialer Ungleichheit in der ökonomischen Sphäre und formeller rechtlicher Gleichheit in der politischen Sphäre? Oder mit Blanke (S. $121 \mathrm{f}$.) gefragt:

"Wie ist es also möglich, daß dieser Widerspruch nicht zur permanenten politischen Krise oder zum revolutionären Zusammenbruch des kapitalistischen Systems führt? Welche objektiven Formen der Mystifikation der auf Ungleichheit beruhenden gesellschaftlichen Verhältnisse bilden sich in der 'Politik' aus, die die Aufrechterhaltung des kapitalistischen Gesellschaftssystems auf der Basis der Tolerierung oder auch der Zustimmung der großen Mehrheit möglich machen?

Es ist die kapitalistische Prduktionsweise selbst, die durch die Warenproduktion und das Kapitalverhältnis Fetischisierungen und Mystifikationen hervorbringt, infolge derer sich die Menschen als gleichartige Einkommensbezieher wähnen, die miteinander um den größtmöglichen Anteil am Wertprodukt kämpfen. Dadurch wird der Klassenantagonismus auf einen Verteilungskonflikt reduziert, der auf dem Wege der Kompromißbildung (z.B. über die 
Höhe der Löhne, der Steuern, der Sozialleistungen, der Subventionen) lösbar ist. Die Kompromißbildung erfolgt in der politischen Sphäre und setzt das Einvernehmen der Beteiligten über die Verfahrensgrundsätze der Kompromißbildung und über die letztinstanzliche Entscheidungsbefugnis des Staates, also einen Verfassungskonsens, voraus. Die in der kapitalistischen Produktionsweise selbst angelegte Möglichkeit eines derartigen Konsenses kann zur historischen Realität werden, wenn die impliziten ideologischen Integrationskräfte durch für alle Beteiligten subjektiv annehmbare Arbeits-, Produktions-, Verwertungs- und Lebensverhältnisse ergänzt werden. Ökonomische Prosperität mindert soziale Konflikte und fördert die Konsensbereitschaft. Die Reduktion des Antagonismus der Klassen auf den Pluralismus der Interessen ist mithin im Kapitalismus möglich, wenn spezifische ökonomisch-soziale und ideologisch-kulturelle Voraussetzungen gegeben sind.

Wegen der widersprüchlichen und tendenziell krisenhaften Dynamik kapitalistischer Verwertung ist ökonomische Prosperität allerdings keine selbstverständliche Begleiterscheinung dieses Systems. Konkurrenz, Rationalisierung, Weltmarktabhängigkeit, Überproduktion, Arbeitslosigkeit usw. erzeugen auch immer wieder verschärfte Interessengegensätze und politische Desintegration. Mit wachsender Krisenhaftigkeit schwinden die materiellen Ressourcen für den sozialen Interessenausgleich, und der kapitalistische Herrschaftskonflikt droht aufzubrechen. Jenseits der Ökonomie-immanenten Fetischisierungs- und Mystifikationstendenzen bedarf es also zusätzlicher, außerökonomischer Krisenstrategien, die den Verfassungskonsens sichern bzw. seine Auflösung verhindern. Unter dem Eindruck des Scheiternsder ersten deutschen Demokratie, der Weimarer Republik, ist gerade in der Bundesrepublik die Gesellschaft ohne Verfassungskonsens ein Trauma.

Der Sicherung dieses Konsenses dienen vor allem zwei Maßnahmen: Durch staatliche Sozialpolitik soll der kapitalismusbedingten ungleichen Verteilung von Einkommen und Lebenschancen die Härten genommen und Fürsorge für Notleidende gewährleistet werden. Vorbeugender Staats- und Verfassungsschutz zielt auf die rechtzeitige Identifizierung und Bestrafung der Gegner der bestehenden staatlichen Ordnung. Darüberhinaus erbringen (vor allem durch den Ausbildungssektor und die Massenmedien laufend reproduzierte) Werte und Normen wie Antikommunismus, Fleiß, Konsum, Pünktlichkeit, Ordnung, Gehorsam gegenüber dem Staat unverzichtbare Integrationsleistungen.

Das Ziel kapitalistischer Integration beschränkt sich nicht auf einen formalen Verfassungskonsens. Die Stabilität der sozialen und politischen Verhältnisse erhöht sich in dem Ausmaß, wie Einvernehmen über essentielle Fragen der Innen- und Außenpolitik in der Gesellschaft besteht. Wenn der gesellschaftliche Konsens auch inhaltliche Vorgaben für wichtige Politikbereiche einschließt, sprechen wir von einem Basiskonsens. Wie in der ersten Folge dieser Artikelreihe ausgeführt wurde (PROKLA 25, S. 18 ff.), beruht die enorme Integrationsfähigkeit der politischen Ordnung der Bundesrepublik darauf, daß sich hier bis zum Ende der fünfziger Jahre ein derartiger Basiskonsens herausbilden konnte.

\subsection{Die Partei als Integrationsinstrument}

In Kontext ökonomisch-sozialer, politisch-rechtlicher und ideologisch-kultureller Integrationsfaktoren ist hier besonders auf den normativen Gehalt von Parteibegriffen zu verweisen, auf Funktions- und Aufgabenzuweisungen also nach dem politischen Selbstverständnis der Parteien. Praktische Relevanz haben vor allem das liberale Parteikonzept und die reformistische Variante des sozialistischen Parteikonzepts erhalten, die dann beide in das Konzept der Volkspartei eingeflossen sind (Stöss, S. 57 ff.). 
Als Kampfinstrument des demokratischen Bürgertums gegen den feudalabsolutistischen Staat spielte die Partei in der zweiten Hälfte des 19. Jahrhunderts nur eine vorübergehende Rolle. Denn nach dem Scheitern der Revolution von 1848 ließ sich das Bürgertum auf den Klassenkompromiß der konstitutionellen Monarchie ein. Das liberale Parteikonzept ordnete sich dem Konstitutionalismus unter und wies den Parteien nun neben der Hauptaufgabe der $R e$ präsentation von ökonomisch-sozialen Interessen und politischen bzw. verfassungsrechtlichen Ordnungsvorstellungen auch den Interessenausgleich und die Konsensbildung zu. In der Weimarer liberalen Staatslehre trat die Integrationsfunktion gleichberechtigt neben die Repräsentationsfunktion: Parteien sollten Vermittlungsagenturen zwischen Klassengesellschaft und Staat sein, mit dem Ziel, aus den unterschiedlichen Interessen einen Gemeinschaftswillen herauszubilden. Dies bedeutete de facto, daß sie im Konfliktfalle gehalten waren, die Gemeinschaftswillensbildung über das Parteiinteresse zu stellen.

Das sozialistische Parteikonzept in der Version etwa von Kautsky wies der SPD eine doppelte und zugleich widersprüchliche Aufgabe zu: Sie sollte die Interessen der Arbeitermassen vertreten und zugleich Avantgarde im Kampf um eine demokratische und sozialistische Gesellschaft sein. Gegen die revolutionäre Klassenpartei setzte Bernstein das Konzept der reformistischen Klassenpartei. Er glaubte an das friedliche Hineinwachsen des Kapitalismus in den Sozialismus, weil das Proletariat auf der Grundlage des(erst noch zu erkämpfenden) allgemeinen Wahlrechts die Herrschaft auf demokratischem Wege erlangen könne. Nach Bernstein ist die Klassengesellschaft nicht allein durch den Antagonismus der Klasseninteressen, sondern zugleich durch das allen Klassen gemeinsame Nationalinteresse geprägt. Der Arbeiter sei nicht nur Mitglied seiner Klasse sondern auch Mitglied der Nation, das Proletariat sei nicht nur Klasse sondern zugleich auch Teil der Nation. Der Partei fällt mithin die Aufgabe $\mathrm{zu}$, die Interessen der Klasse zu vertreten und sie in das Nationalinteresse einzufügen. Demokratie ist nach Bernstein daher die Hochschule des Kompromisses und bedeutet Milderung der Klassengegensätze. Im Grunde genommen war damit der weitere Weg des sozialdemokratisch-reformistischen Selbstverständnisses zur »Volkspartei« vorgezeichnet. Unter dem unmittelbaren Eindruck der Auflösung der Weimarer Republik fand Sigmund Neumann (S. 16, $18,19)$ folgende Formulierung für die Aufgabe der Parteien, die offenbar als drohende Warnung vor der Klassenpartei verstanden werden sollte:

»Nur wenn eine gemeinsame Grundlage für die auf spezifische Wünsche ausgerichteten Parteien besteht, wird der politische Kampf nicht zum Auseinanderbrechen der Gesamheit führen. Nur wenn es Entscheidendes gibt, das eint, kann Trennendes ausgeglichen werden, sind selbst Opfer, Verzicht und Unterliegen tragbar....

Diese Verflechtung der Parteien mit dem Schicksal der Gesamtheit, von der sie ein Teil sind, ergibt sich auch aus ihrem 'politischen' Charakter. Politisch aber werden sie nicht nur durch ihren Machtanspruch, sondern durch Erfüllung ihrer wesentlich politischen Funktion. Sie beruht nicht nur auf der Willenskonzentration der chaotisch differenzierten Einzelwillen, sondern auf der Durchpolitisierung solcher privater Interessenbestrebungen und ihrer Einbettung der Gesamtinteressen.

Das bedeutet aber nichts anderes als eine Verwandlung des einzelnen und seiner Interessen selbst, ihn zum Zoon politicon machen oder - wie es in moderner Terminologie heißt - ihn in die Gesamtheit hineinintegrieren ... Hier setzt die eminent politische Aufgabe der Partei und ihrer Führung gegenüber ihrer Anhängerschaft ein, die sie aus dieser politischen Gesinnung heraus zu Opfern zwingen wird.« 


\subsection{Das Konzept der»Volkspartei«}

Mit der Wieder- bzw. Neugründung politischer Parteien nach der Zerschlagung des Nationalsozialismus begann sich auch das Selbstverständnis der Parteien zu verändern. Sie wurden im Grundgesetz als Bestandteile des Verfassungslebens institutionalisiert, und es bestand unter den führenden Vertretern des politischen Katholizismus, des Liberalismus und der Sozialdemokratie Einvernehmen darüber, daß die Aufgabe der Parteien im Rahmen des Verfassungskonsenses nicht nur darin besteht, unterschiedliche Traditionen, Interessen und Zielsetzungen zu organisieren und in den politischen Willensbildungsprozeß einzubringen. Parteien sollten darüber hinaus das Zusammenwirken der sozialen und politischen Kräfte im Hinblick auf die Staatswillensbildung gewährleisten. Mit Blick auf die Weimarer Verhältnisse wurde die Integrationsfunktion der Parteien nun als vordringlich bewertet. Im Zuge der Herausbildung eines Basiskonsenses in der westdeutschen Gesellschaft entwickelten sich CDU/CSU, FDP und SPD zunehmend zu staatlichen Integrationsagenturen, die die Funktion der Repräsentation spezifischer Interessen und Bedürfnisse zurückstellen hinter die Integration ihrer Basis in die bestehende Ordnung und die Legitimation staatlicher Tätigkeit. Wir haben die drei staatstragenden Parteien deshalb als demokratische »Massenlegitimationsparteien « bezeichnet. Ihrem Selbstverständnis nach sind sie »Volksparteien«, die vor allem das politische Führungspersonal herausbilden und miteinander bei Wahlen um die Besetzung politischer Ämter konkurrieren. Kaste/Raschke (S. 30 f.) beschreiben die Volksparteien u.a. so: Die Volkspartei (VP)

»- sieht die Massen ausschließlich oder primär als Objekt der Wahlmobilisierung,

- verschafft ihren Funktionsträgern im Staatsapparat eine hohe Autonomie gegenüber möglichen 'Einmischungen' der Massen und Parteimitglieder im politischen Entscheidungsprozeß,

- versucht, gesellschaftlichen Wandel primär in Gestalt von Anpassungsreform zu bewältigen,

- steht permanent und prinzipiell in Widersprüchen zwischen ihrem Legitimationsprogramm und ihrer realen Interessenvertretungs- und Steuerungsfunktion,

- ist durch eine ganz überwiegend individuelle Organisationsweise bestimmt.

Dazu treten zwei interdependente, grundlegende Merkmale, die häufig für sich als Definitionsmerkmale der VP gelten:

Die VP hat

- eine breite, sozialstrukturell heterogene Wählerbasis,

- ein für jeweilige Situationen und Gruppen anpassungsfähiges Programm, das primär der Integration vieler Tendenzen dient."

Damit zielt der gegenwärtig im Parteiensystem der Bundesrepublik vorherrschende Parteityp auch seinem Selbstverständnis nach in erster Linie auf die Stabilität und Funktionsfähigkeit des Staates. Die Massenlegitimationsparteien stellen fast schon Staatsparteien dar, die ihre soziale Basis für den Staat funktionalisieren (wollen). Freilich: Hierbei handelt es sich um einen speziellen Parteityp, der gegenwärtig die Szene beherrscht. Dieser Typ darf keinesfalls verabsolutiert, als die Partei schlechthin mißverstanden werden.

\subsection{Parteiensystem und Sozialstruktur}

Mit zunehmender Konsenshaftigkeit bildet ein Parteiensystem den Klassencharakter der Gesellschaft nur noch indirekt ab. Infolge der Reduktion des Klassenantagonismus auf einen 
Verteilungskonflikt bestimmen vorrangig Beschäftigungsverhältnisse, Einkommensarten und Berufsgruppen die sozialen Konfliktlinien innerhalb des Parteiensystems sowie die Parteibindungen und das Wahlverhalten der Bevölkerung. Hinzu kommen politische Traditionen lebensgeschichtlicher wie regionaler Art, Lagermentalitäten und Milieubindungen. Als wesentliche Determinanten des Wahlverhaltens gelten die Zugehörigkeit zu den Berufsgruppen Arbeiter, Angestellte und Beamte (als »Zwischenschichten«) sowie Selbständige (einschl. Landwirte) und die Bindungen an spezifische sozio-ökonomische und sozio-kulturelle

Tabelle 1: Mitgliederentwicklung der Parteien in der Bundesrepublik

\begin{tabular}{rrrrrrrr}
\hline Jahr & CDU & CSU & FDP & SPD & KPD/DKP & SRP/DRP/NPD & $\begin{array}{c}\text { Die } \\
\text { Grünen }\end{array}$ \\
\hline 1947 & 400000 & 82189 & & 875479 & $324000(\mathrm{KPD})$ & & \\
1951 & & & 82890 & 649529 & $148000(\mathrm{KPD})$ & $10300 *(\mathrm{SRP})$ & \\
1952 & 200000 & & & 627817 & $120000(\mathrm{KPD})$ & & \\
1956 & 245000 & 43500 & & 612219 & $78000 *(\mathrm{KPD})$ & $4000 *(\mathrm{DRP})$ & \\
1959 & & & 80000 & 634254 & & & \\
1963 & 250000 & 56018 & & 648415 & & $4500(\mathrm{DRP})$ & \\
1968 & 286541 & 73618 & 57034 & 732446 & $9085(\mathrm{DKP})$ & $27000(\mathrm{NPD})$ & \\
1970 & 329293 & 76655 & 56531 & 820202 & $30000(\mathrm{DKP})$ & $21000(\mathrm{NPD})$ & \\
1973 & 457393 & 111913 & 63205 & 973601 & $39344(\mathrm{DKP})$ & $12000(\mathrm{NPD})$ & \\
1977 & 664214 & 159475 & 79539 & 1006316 & $42500(\mathrm{DKP})$ & $9000(\mathrm{NPD})$ & \\
1981 & 705116 & 175273 & 86931 & 956490 & $48856(\mathrm{DKP})$ & $6600(\mathrm{NPD})$ & 15352 \\
1982 & 718889 & 178523 & 79000 & 926070 & $50000(\mathrm{DKP})$ & $6000(\mathrm{NPD})$ & 20000 \\
1983 & 734555 & 185428 & 71456 & 925630 & & & $30000 *$ \\
\hline
\end{tabular}

Quellen: Eigene Angaben der Parteien, zumeist Jahresende; NPD: BMI.

* Begründete Schätzung.

Tabelle 2: Organisationsgrad der Parteien in der Bundesrepublik (1983/84)

\begin{tabular}{lrccc}
\hline & Wähler & Mitglieder $^{2}$ & Relation & Organisationsgrad \\
\hline Bundesgebiet & & & & \\
& & & & \\
Grüne & 2167431 & 25000 & $87: 1$ & $1,2 \%$ \\
SPD & 14865807 & 950000 & $16: 1$ & $6,4 \%$ \\
CDU & 14857680 & 734082 & $20: 1$ & $4,9 \%$ \\
CSU & 4140865 & 182665 & $23: 1$ & $4,4 \%$ \\
FDP & 2706942 & 78763 & $34: 1$ & $2,9 \%$ \\
\hline
\end{tabular}

1 Jeweils Zweitstimr in.

2 Zu den Bundesparteien s. FAZ vom 22.7.1983; FDP lt. Mitteilung an den Verf. v. 12.7.1983; Mitgliederzahlen der Grünen It. Mitteilung an den Verf. Apr.-Sept. 1983 u. SZ v. 24.11.1983.

Quelle: Fogt, PVS 1984, S. 105. 
Milieus, insbesondere an die Gewerkscbaftsbewegung und an cbristlich-religiöse, insbes. katholische Milieus (zum konfessionellen Schisma s. PROKLA 25, S. 19 ff.). Daraus ergeben sich nun keine streng gegeneinander abgegrenzten Wählersegmente, aber doch deutliche Affinitäten zu den beiden großen (zum Organisationsgrad: Tabelle 2) Parteien CDU/CSU und SPD. Während letztere sich vor allem auf die Arbeiter und die gewerkschaftlich organisierten Beamten und Angestellten stützt, speisen sich die Wahlerfolge der Union hauptsächlich aus dem katholischen Milieu, aus der Gruppe der Selbständigen und aus den gewerkschaftlich ungebundenen Angestellten und Beamten. Da etwa drei Viertel der Wähler von CDU/ CSU und SPD "Stammwähler « sind (bei der Bundestagswahl 1983 waren es weit über $80 \%$ : Forschungsgruppe Wablen, Bundestagswahl 1983, S. 8), also immer dieselbe Partei wählen, unterliegen beide Parteien stabilen und voneinander abgrenzbaren sozialen Bindungen. Die SPD ist durch die Arbeiter und die »kleinen« Angestellten und Beamten geprägt, die Union durch die Selbständigen und die »höheren « Angestellten und Beamten. Bei der FDP liegt der Anteil der "Stammwähler« bei etwa $40 \%$, hinsichtlich der Grünen/Alternativen kann man von einer "Stammwählerschaft« noch nicht sprechen.

Bei Wahlkämpfen geht es heute also vordringlich um die Gewinnung von Wechselwählern. Diese machen zwar nur einen Anteil von $20 \%$ bis $25 \%$ an der Wählerschaft der beiden groBen Parteien aus, entscheiden aber letztendlich das Wahlergebnis. Da den Parteien die "Stammwähler mehr oder weniger sicher sind, orientieren sie sich bei der Imagepflege, bei der Formulierung von Programmen und in der praktischen Politik nicht oder doch nur kaum an ihrer angestammten Basis, sondern an dem Bevölkerungsteil, der weder dem gewerkschaftlichen noch dem christlich-katholischen Milieu zuzurechnen ist, berufsmäßig überwiegend in der Mittelschicht (Angestellte, Beamte) angesiedelt ist und seine W/ahlentscheidung jeweils von aktuellen Einflüssen (Beliebtheit der Spitzenkandidaten, Problemlösungsfähigkeit der Parteien etc.) abhängig macht. (Aus diesem Potential speisen sich überwiegend auch die Grünen/Alternativen.) Dieser "Trend zur Mitte«, der durch den Wandel der Sozialstruktur (vgl. dazu auch Feist/Liepelt 1976) und durch die Herausbildung des Basiskonsenses gefördert wird, korrespondiert mit der Rolle der Massenlegitimationsparteien und ihrem Selbstverständnis als bei Wahlen miteinander um die Besetzung politischer Positionen wetteifernden »Volksparteien«. Angesichts der jeweils spezifischen sozialen Verankerung von SPD und CDU/CSU, die sich deutlich auch in der Berufsstruktur ihrer Mitglieder (Tabellen 3 bis 6) niederschlägt, kann die Vernachlässigung der Interessenvertret ung der jeweiligen Basis zugunsten der Überbetonung der Legitimationsfunktion und einer taktischen Hinwendung zu fluktuierendem Wählerpotential aus den Mittelschichten jedoch zu einem Repräsentationsdefizit führen, mit der Folge, daß "Stammwähler« demobilisiert werden. Dieser Effekt scheint in gewissem Umfang für die Stimmverluste der SPD bei den vergangenen Wahlen mitverantwortlich zu sein.

Vergleicht man die Berufsstruktur der Mitgliederschaften der vier großen Parteien CDU, CSU, FDP und SPD, dann zeigt sich, daß die soziale Integrationsfunktion des Parteiensystems arbeitsteilig, also mit spezifischen Schwerpunkten bei den vier Parteien erfolgt. Die von Gluchoweski/Veen und Veen/Gluchowski dargestellten Nivellierungstendenzen sind wohl in erster Linie der Gesamtveränderung der Sozialstruktur und der sozialstrukturellen Öffnung der SPD nach 1958/60 anzulasten. Bei der Interpretation der Tabellen 3 bis 6 ist nicht nur zu berücksichtigen, daß sich zwischen 1950 und 1981 unter den Erwerbspersonen der Anteil der Arbeiter von $51 \%$ auf $42 \%$, der der Selbständigen von $28 \%$ auf $12 \%$ vermindert und der der Angestellten und Beamten von $21 \%$ auf $46 \%$ erhöht hat. Die detaillierte 
Aufschlüsselung der Gesamtbevölkerung nach Berufsgruppen für das Jahr 1977 ergibt folgendes Bild (i.v.H.).

$\begin{array}{lrlr}\text { Arbeiter } & 22 & \text { Landwirte } & 3 \\ \text { Angestellte } & 19 & \text { Rentner } & 19 \\ \text { Beamte } & 4 & \text { Hausfrauen } & 22 \\ \text { Selbständige } & 7 & \text { in Ausbildung } & 7\end{array}$

Sie verweist auf deutliche Differenzen zwischen den Parteien und, unter Berüicksichtigung zusätzlichen Datenmaterials (Schmollinger/Stöss, Schmollinger), auf spezifische Disparitäten. Die Arbeiter sind in den bürgerlichen Parteien deutlich unterdurchschnittlich repräsentiert. Trotz des abnehmenden Arbeiteranteils an den SPD-Mitgliedern seit 1950 ist die Arbeiter-

Tabelle 3: Berufliche Gliederung der CDU-Mitglieder 1976 und 1984 (i.v.H.)

\begin{tabular}{lcc}
\hline & $1976(30.6 .)^{1}$ & $1984(30.4)^{2}$ \\
\hline Arbeiter & 10,9 & 10,2 \\
Angestellte & 27,2 & 28,0 \\
Beamte & 12,5 & 12,3 \\
Selbständige & 26,4 & 24,5 \\
Helfer in Familienbetrieben & 0,4 & 0,4 \\
Rentner & 5,3 & 4,8 \\
Hausfrauen & 9,8 & 11,1 \\
in Ausbildung & 5,8 & 6,5 \\
Arbeitslose & 0,1 & 0,4 \\
ohne Angaben & 1,5 & 1,8 \\
\hline
\end{tabular}

1 CDU, Statistischer Bericht der zentralen Mitgliederkartei, Stand: 30.6.1976.

2 CDU, Bericht der Bundesgeschäftsstelle. Anlage zum Bericht des Generalsekretärs. 32. Bundesparteitag, 8.-11. Mai 1984, Stuttgart, Bonn, im Mai 1984, S. 52.

Tabelle 4: Berufliche Gliederung der CSU-Mitglieder 1978 und 1983 (i.v.H.)

$\left.\begin{array}{lcc}\hline & 1978(31.12 .) & 1983(31.12 .) \\ \hline \text { Arbeiter } & 14,5 & 14,7 \\ \text { Angestellte } & 21,0 & 22,7 \\ \text { Beamte } & 12,2 & 12,5 \\ \text { Landwirte } & 17,7 & 10,3 \\ \text { Selbständige } & 10,6 \quad 30,8 & 15,5 \\ \text { Freiberufliche } & 2,5 & 2,3\end{array}\right\} 28,1$

Quelle: CSU, Zentralkartei, Mitgliederstruktur; Stand 31.12.1978 und 31.12.1983. 


\begin{tabular}{lc}
\hline Arbeiter/Facharbeiter & 5 \\
Angestellte & 30 \\
Beamte & 14 \\
Selbständige/Freie Berufe & 19 \\
Rentner & 12 \\
Hausfrauen & 11 \\
in Ausbildung & 9 \\
\hline
\end{tabular}

Quelle: FDP-Mitgliederbefragung, Feb. 1977.

Tabelle 6: Berufliche Gliederung der SPD-Mitglieder 1977 und 1981 (i.v.H.)

\begin{tabular}{lcc}
\hline & Umfrage & Schätzung \\
& 1977 & 1981 \\
\hline Arbeiter & 22 & 21 \\
Angestellte & 26 & 27 \\
Bearnte & 13 & 14 \\
Selbständige & 5 & 5 \\
Schüler/Lehrlinge/Studenten & 5 & 4 \\
Rentner/Pensionäre & 18 & 17 \\
Hausfrauen & 7 & 8 \\
Sonstige/K.A. & 4 & 4 \\
\hline
\end{tabular}

Quelle: Horst Becker, Bodo Hombach u.a., Die SPD von innen, Bonn 1983, S. 59

schaft in der Sozialdemokratie immer noch durchschnittlich vertreten. Selbst wenn man annimmt, daß ein erheblicher Teil der Rentner und Hausfrauen der Arbeiterklasse zuzurechnen ist, dürfte der Arbeiteranteil bei $40 \%$ liegen und etwa dem Anteil an den Erwerbspersonen entsprechen. Von einer überproportionalen Repräsentanz kann daher nicht mehr die Rede sein. Selbst wenn die Arbeiter die größte Berufsgruppe innerhalb der SPD-Mitglieder darstellen, sinkt ihre innerparteiliche Bedeutung in der Hierarchie der Amtsinhaber. In innerparteilichen Spitzenpositionen und unter den Parlamentariern der Partei sind sie kaum vertreten.

Die Selbständigen sind in allen bürgerlichen Parteien stark überrepräsentiert. Da die FDP seit ihrem politisch-programmatischen Kurswechsel gegen Ende der sechziger Jahre hier viel Terrain verloren hat (1965 betrug der Anteil der Selbständigen an ihren Mitgliedern noch $31 \%$ ), stellen die Unionsparteien heute die hegemoniale Kraft innerhalb des Besitzbürgertums dar. Überrepräsentiert sind auch die Angestellten und vor allem die Beamten, und zwar mehr oder weniger in allen Parteien. Vor allem die FDP findet beiden Angestellten/Beamten ohne feste Parteibindung mittlerweile ihr wichtigstes Einzugsgebiet. Mit Blick auf die Führungspositionen und die parlamentarische Repräsentanz der Parteien nehmen die Angestellten und Beamten im Öffentlichen Dienst in allen Parteien eine dominierende Stellung ein, womit die Tendenz zur »Verstaatlichung« der Parteien auch ihren sozialstrukturellen Ausdruck findet (Schmollinger, S. 22). 
Tabelle 7: Frasenanteile an den Mitgliederschaften von SPD, CDU, CSU, und FDP (i.v.H.)

\begin{tabular}{lcccccccc}
\hline & \multicolumn{2}{c}{ SPD } & \multicolumn{2}{c}{ CDU } & \multicolumn{2}{c}{ CSU } & \multicolumn{2}{c}{ FDP } \\
& 1977 & 1983 & 1977 & 1984 & 1978 & 1983 & 1976 & 1981 \\
\hline weiblich & 21,7 & 24,5 & 20,2 & 21,7 & 12,8 & 13,7 & 19 & 25 \\
männlich & 78,3 & 75,5 & 79,8 & 78,3 & 87,2 & 86,3 & 81 & 75 \\
\hline
\end{tabular}

Quelle: Angaben der Organisationsabteilungen der Parteien.

Besonderer Aufmerksamkeit muß auch der unzureichenden Vertretung von Frauen in den Parteien gelten (Tabelle 7). Sieht man einmal von der in dieser Hinsicht besonders defizitären CSU ab, dann beträgt der Frauenanteil unter den Parteimitgliedern gegenwärtig etwa ein Fünftel bis ein Viertel (SPD, FDP). Jedoch verhält es sich hier wie bei den Arbeitern: Innerhalb der organisatorischen Hierarchien der Parteien schwindet der Fraueneinfluß zunehmend. Parteipolitik ist an entscheidender Stelle ausschließlich Männersache, Angelegenheit männlicher Polit-Profis mit entsprechender Karriere ( $\leadsto$ Ochsentour $«)$, die aus politiknahen Berufen (Öffentlicher Dienst, Verbände, Wirtschaft) stammen und ein etatistisches "KastenBewußtsein« (positive Rollenidentifikation, Orientierung auf Gruppenkonsens und Konfliktvermeidung) ausgebildet haben (vgl. auch Herzog), was jedoch der Legitimations- und Integrationsfunktion der politischen Parteien außerordentlich dienlich ist.

Der gegenwärtig im westdeutschen Parteiensystem dominierende Parteientyp der Massenlegitimationspartei unterliegt als Organisationsform im Prozeß der Vermittlung von ÖKonomie und Politik dem Widerspruch von spezifischer sozialer Bindung und Staatsorientierung. Indem die Massenlegitimationspartei als Vermittlungsagentur zwischen Klassengesellschaft und Staat fungiert und auch fungieren will und dabei vor allem mit Blick auf die Stabilität und Funktionsfähigkeit des Staates das Zusammenwirken der sozialen und politischen Kräfte mit dem Ziel einer effizienten Staatswillensbildung anstrebt, gerät sie in den Konflikt zwischen Interessenrepräsentationsfunktion einerseits und Legitimationsfunktion andererseits. Dieser Konflikt wurde spätestens seit Bestehen des Basiskonsenses stets zugunsten der Legitimationsfunktion gelöst, bewirkte aber auch, jeweils nach der interessenmäßigen und politischen Orientierung der Parteibasis, Spannungen, Repräsentationsdefizite und Demobilisierungseffekte.

In Wirtschaftskrisen kann die Verschärfung des Verteilungskampfs dazu führen, daß sich der Konflikt zwischen Interessenrepräsentation und Legitimation zu einem Widerspruch ausweitet, zumal dann in der Regel auch die Ressourcen für eine konsensstiftende staatliche Sozialpolitik schmelzen. Durch ihre spezifischen sozialen Bindungen geraten CDU/CSU und SPD nämlich rasch unter den Druck ihrer Basis. Der Bedarf an nachhaltiger Interessenvertretung steigt, während gleichzeitig auch der Legitimationsbedarf des Staates wächst. Unter diesem Widerspruch leidet besonders die Partei, deren Anhänger von der kapitalismusbedingten ungleichen Verteilung von Einkommen und Lebenschancen am meisten betroffen. sind, hierzulande also die SPD. Nimmt sie sich konsequent den Belangen ihrer Basis an, dann ist damit ihr Selbstverständnis als » Volkspartei« in Frage gestellt. Verhält sie sich dagegen weiterhin als staatliche Legitimations- und Integrationsagentur, dann demobilisiert sie inre Anhänger und verprellt ihre »Stammwähler«. 


\subsection{Die Grïnen/Alternativen als Anti-Massenlegitimationsparteien}

Die Grünen benutzen gelegentlich die Bezeichnung»Anci-Parteien-Partei«(Petra Kelly) zur Selbstdarstellung. Dies trifft, bislang jedenfalls, im großen und ganzen auf die Binnenstruktur und die Arbeitsweise der Grünen und Alternativen zu. Die Vernetzung mit den »Neuen Sozialen Bewegungen«, die basisdemokratische innere Ordnung, das Öffentlichkeitsprinzip, das imperative Mandat und das Rotationsprinzip richten sich gerade gegen den Typ der Massenlegitimationspartei. Ihre sozialstrukturelle Selektivität, die mangelnde Berücksichtigung von Interessen und politischen Zielen der Basis, die unzureichenden innerparteilichen Partizipationsmöglichkeiten und schließlich die Verselbständigung der Führungsgruppen haben wesentlich zur Entstehung der Bürgerinitiativen, der "Neuen Sozialen Bewegungen« und schließlich der Grünen/Alternativen beigetragen. Diese sind mithin in erster Linie als der parteiförmige Ausdruck der - in den siebziger Jahren als Folge der autoritär-etatistischen Demokratieverfassung der Bundesrepublik explosionsartig angestiegenen - Partizipationsbedürfnisse zu bewerten. Dabei gilt Partizipation nicht als Selbstzweck sondern als Mittel zur aussichtsreicheren Durchsetzung von politischen Zielen, deren Bedeutung von den Massenlegitimationsparteien nicht erkannt oder wenigstens doch unterbewertet worden ist bzw. die sich mit den vorherrschenden und mit dem Basiskonsens korrespondierenden Politikprioritäten nicht oder nur schwer vereinbaren ließen.

Das Parteiensystem ist damit gegenwärtig einer zweifachen Belastungsprobe ausgesetzt: nämlich dem aus dem kapitalistischen Verteilungskonflikt erwachsenden erhöhten Interessendruck einerseits und den aus den Legitimationsdefiziten in der politischen Sphäre resultierenden Partizipationsanforderungen andererseits. Beide Faktoren haben zusammengenommen 1982 den Regierungswechsel ermöglicht.

\section{Die Entwicklung des Parteiensystems bis zur Bundestagswahl 1976}

\subsection{Die Vollendung des Basiskonsenses}

Ausgehend von der Frage nach den Ursachen für die enorme Stabilität des westdeutschen Parteiensystems haben wir in den ersten beiden Folgen dieses Artikels am Beispiel der Bundestagswahlen die wachsende Integrationsfähigkeit des politischen Systems aufgezeigt. Ihm liegt ein Basiskonsens zugrunde, der über einen formal-rechtlichen Konsens bezüglich der freien Verfügung über das Privateigentum sowie der Verfahrensregeln in der politischen Sphäre hinaus auch das Einvernehmen über wesentliche ideologische, ökonomische und politische Grundlagen der westdeutschen Gesellschaft (Antikommunismus, Westintegration, Landesverteidigung, Marktwirtschaft, Sozialordnung) umfaßt. Die Herausbildung dieses Konsenses vollzog sich innerhalb des Parteiensystems zunächst auf dem Wege der Entstehung und Festigung eines bürgerlichen Intraklassenkonsenses (quantitative Ausweitung der sozialen Basis der Bürgerblock-Parteien CDU/CSU, FDP, DP, BHE und Dominanz der Unionsparteien innerhalb des Bürgerblocks) und schließlich durch die programmatische Anpassung der Sozialdemokratie - sie verfügte bereits seit Anfang der fünfziger Jahre über eine hegemoniale Position innerhalb der Arbeiterklasse - an die innenpolitischen und außenpolitischen Grundlagen des »CDU-Staats«.

Mit der Vollendung des Basiskonsenses 1958/60 verlor auch der Blockgegensatz zwischen 
den bürgerlichen Parteien und der SPD an Schärfe und Bedeutung. Parallel zur Milderung der politischen Polarisierung vollzog sich eine Abschwächung der sozialstrukturellen Polarisierung. Die Anpassung ermöglichte es der SPD, in bürgerliche Wählerschichten einzudringen und damit aus ihrem $» 30$-Prozent-Turm« (Zweitstimmenanteil bei den ersten drei Bundestagswahlen) auszubrechen. Anläßlich der Bundestagswahl 1961 fanden erstmals in größerem Umfang Wählerwanderungen zwischen den Bürgerblock-Parteien und der SPD statt, deren soziale Basis sich nun qualitativ und quantitativ auszuweiten begann.

Mit dieser Bundestagswahl hatte sich auf Bundesebene ein "Drei-Parteien-System « etabliert, die kleinen Bürgerblock-Parteien waren bundesweit fast vollkommen bedeutungslos geworden, und auch antikapitalistische und antidemokratische (neofaschistische) Alternativen blieben im Parteiensystem angesichts der Integrationskraft des Basiskonsenses ohne Erfolgsaussichten.

\subsection{Die Auflösung des Bürgerblocks und die Bildung der sozialliberalen Koalition}

Die Auflösung des Bürgerblocks im Jahre 1966 markierte das Ende des »CDU-Staats«. Ihm versetzte die erste größere Wirtschaftskrise (1966/67) den Todesstoß, nachdem es den Unionsparteien seit Anfang der sechziger Jahre nicht mehr gelungen war, dem funktionalen Imperativ des politischen Systems hinsichtlich der notwendigen Steuerungs-, Anpassungsund Modernisierungsleistungen hinreichend Rechnung zu tragen. Das Scheitern des Bürgerblocks war also weniger durch die Beendigung des Blockgegensatzes, sondern in erster Linie durch politisches Versagen vor den veränderten innenpolitischen und außenpolitischen Gegebenheiten bedingt. So erwiesen sich CDU und CSU außerstande, die internationalen Entspannungstendenzen durch eine rechtzeitige und konsequente Revision ihrer auf Konfrontation und roll back zielenden Ostpolitik nachzuvollziehen. Zudem machten die Strukturkrisen einzelner Branchen (Kohle, Stahl, Textil), die unzureichenden wissenschaftlich-technischen Voraussetzungen für die notwendige Produktivitätssteigerung infolge der Vollbeschäftigung und der verschärften Weltmarktkonkurrenz ("technologische Lücke«, »Bildungskatastrophe $\ll$ ) und schließlich die Überproduktions- und Finanzkrise 1966 staatliche Interventionen erforderlich, zu denen sich die Union mit ihrer interventionsfeindlichen neoliberalen Wirtschaftspolitik nicht bereit finden mochte. Mit dem $\mathrm{Ab}$ schluß der Konsolidierungsphase der Bundesrepublik standen darüber hinaus eine Vielzahl von Problemen in den Bereichen Finanz- und Steuerpolitik, Sozialpolitik, Bildungs- und Hochschulpolitik und nicht zuletzt Fragen der Mitbestimmung und der Unternehmensverfassung auf der politischen Tagesordnung, wofür die Union ebenfalls keine relevanten Lösungsvorschläge bereit hielt.

Von dem Klima der Reformnotwenigkeit und Reformbereitschaft profitierte jedoch die SPD: Zum einen stieß ihr Konzept einer entspannungsorientierten Ostpolitik auch international auf wachsende Zustimmung, zum anderen versprachen ihre Reformbereitschaft und vor allem ihre keynesianisch fundierte Wirtschaftspolitik die erfolgreiche Lösung der anstehenden Probleme. In der Sprache der Wahlforscher formuliert: Vor allem Beamte und Angestellte (und hier wiederum überdurchschnittlich Katholiken) sowie katholische Arbeiter sprachen der SPD zunehmend die Sachkompetenz zur Lösung der dringenden politischen Fragen zu. Die »geistige Führung«, die politisch-konzeptionelle Hegemonie ging also von der CDU auf die SPD über, die dann auch zunächst in der Großen Koalition und später in der sozialliberalen Koalition die Initiative hatte. 
Der Zerfall des Bürgerblocks war zugleich auch durch wachsende Differenzen zwischen den Unionsparteien und der FDP und durch die Annäherung von FDP und SPD - zunächst vor allem in außenpolitischen Fragen - bedingt. Als die Bundeskoalition 1966 durch die Finanzkrise zerbrach, war ein Zusammengehen von SPD und FDP jedoch noch nicht möglich. Unter den besitzbürgerlichen Liberalen herrschten gerade in wirtschaftspolitischer Hinsicht noch starke Vorbehalte gegenüber der immer noch weithin des Sozialismus verdächtigten SPD. In der Oppositionszeit, während der Großen Koalition, leitete der linksliberale Flügel innerhalb der FDP dann einen programmatischen Reformkurs ein, der 1971 mit der Verabschiedung der »Freiburger Thesen (liberale Reform des Kapitalismus) abgeschlossen war. Der mit einem innerparteilichen Führungswechsel verbundene Kurswechsel brachte die Partei vorübergehend zwar an den Rand des organisatorischen Ruins, es gelang ihr aber bis 1972, die Verluste aus dem »alten« besitzbürgerlichen Mittelstand durch Gewinne bei den Angestellten und Beamten auszugleichen. Diese Schwerpunktverlagerung vom "Alten Mittelstand« zum »Neuen Mittelstand« machte sie politisch-programmatisch wie sozialstrukturell zu einer Partei der reformorientierten »Steuerungskader« (Schiller), womit die Voraussetzungen für die sozialliberale Koalition geschaffen worden waren.

\section{Das Ende der sozialliberalen Koalition}

\subsection{Die Politik der SPD}

Mit großem Engagement und einer Fülle von Initiativen begann die sozialliberale Koalition 1969, ihre Reformpläne in praktische Politik umzusetzen. Daß sie mit ihrer Politik der inneren Reformen, im Gegensatz zur Ostpolitik, nur Teilerfolge erzielte (detailliert Heimann, S. 2098 ff.) und die vielfach überzogenen Hoffnungen derjenigen enttäuschte, die 1969 eine neue Ära hatten anbrechen sehen, lag nicht allein an den durch die kapitalistische Ökonomie vorgegebenen Handlungsgrenzen des Staates. Es fehlte auch an Macht.

Die oppositionelle CDU/CSU besaß im Bund und in den Ländern - der Bundesrat entwickelte sich Ende der siebziger Jahre nachgerade zu einer Blockadeinstanz für die Koalition - wachsenden Einfluß und war in der Lage, viele Reformvorhaben zu verwässern oder zu verschleppen. In vielen Fragen spielte auch die FDP eine Bremserrolle. In außenund wirtschaftspolitischen Reformvorhaben sah sich die Koalition zudem mit einer finanzstarken und publizistisch wirksamen außerparlamentarischen Opposition (»Rechtskartellk) konfrontiert, deren Arm bis in die rechten Flügel von SPD und FDP hineinreichte und die sogar imstande war, Abspaltungen zu initiieren (Nationalliberale Aktion, Bund Freies Deutschland, Aktionsgemeinschaft Vierte Partei). In familienpolitischen Angelegenheiten, in Sonderheit bei der Reform des $\$ 218$ StGB, erwies sich die katholische Kirche als außerordentlich potenter Widersacher, und das Bundesverfassungsgericht blockierte oder veränderte so manches Reformprojekt ( Ersatzgesetzgeber«). Auf Gegenwehr stieß die Politik der inneren Reformen auch im Lager der Unternehmer, soweit diese über die kapitalismusfunktionale Modernisierung der Volkswirtschaft hinaus auch Interessen der Arbeiter bzw. der Gewerkschaften (Humanisierung, Mitbestimmung, Sozialpolitik etc.) berücksichtigte (vgl. a. M.G. Scbmidt 1978). (Siehe auch Tabelle 8, S. 47)

Mit der ökonomischen Krise 1974/75 und der seitdem wachsenden Massenarbeitslosigkeit geriet die SPD immer heftiger in die Defensive. Der Keynesianismus als ökonomische und 
gesellschaftspolitische Grundlage der Reformpolitik (Hoffmann) verlor an Bedeutung. Mit dem Kanzlerwechsel von Brandt zu Schmidt (1974) begann die Ablösung der Reformpolitik durch eine Politik technokratischer Krisenbewältigung und Mängelverwaltung ohne verbindliche programmatische Grundlage. Schmidt wollte »den Staat anständig regieren«, die Krise als Chance der Modernisierung der Volkswirtschaft nutzen und durch angebotsorientierte Investitionshilfen zur Erhöhung der Produktivität und damit zur Verbesserung der Konkurrenzfähigkeit auf dem Weltmarkt beitragen. Dies bedeutete zwangsläufig den weiteren Abbau von Arbeitsplätzen und die Dequalifikation von Arbeitskraft und wirkte sich vor allem zu Lasten der Facharbeiter, einer wichtigen sozialdemokratischen Wählergruppe, aus. Die Haushaltssanierung vollzog sich primär auf dem Wege der Verringerung der Staatsquote. Die angestrebte Reduzierung der Sozialausgaben zielte zudem auf die Entlastung der Unternehmen, deren Investitionsbereitschaft bzw. -fähigkeit durch weitere Abgabeentlastungen und durch Lohnsenkungen gefördert werden sollte. Mit dieser "post-keynesianischen« Krisenstrategie geriet die sozialdemokratische Führung bald nicht nur in heftige Konflikte mit der eigenen Partei und mit den Gewerkschaften, sondern auch unter Beschuß der Unionsparteien und - verhaltener - der FDP, weil sie mit Rücksicht auf ihre soziale Basis stets bemüht war, allzu harte Krisenfolgen abzufedern.

Aber nicht nur ihre Wirtschafts- und Sozialpolitik manövrierte die SPD in innere und äußere Schwierigkeiten. Der Vorrang industrieller Wachstumspolitik zu Lasten der Umwelt, das rigide vollzogene Atomprogramm, der autoritär-etatistische Regierungsstil und schließlich die harte polizeistaatliche Repression außerparlamentarischer Aktivitäten beförderten die Ausbreitung der Ökologie- und Alternativbewegung, die zunehmend Resonanz auch in Teilen der SPD hatte. Das starre Festhalten an der Aufrüstung der Nato mit Atomraketen provozierte schließlich die Entstehung einer massenhaften Friedensbewegung, mit der sich ebenfalls große Teile der SPD solidarisierten. Endlich trug auch die Veränderung des durch die Wirtschaftskrise und durch wachsende innere wie äußere Polarisierung gekennzeichneten politischen Klimas dazu bei, daß sich die Sozialdemokratie Anfang der achtziger Jahre auf dem Tiefpunkt ihrer Wählerresonanz befand: Das für ein Krisenklima typische Status quoDenken, die Gemeinschaftsrhetorik, das Bedürfnis nach Harmonie, die weit verbreitete Überzeugung »wir sitzen alle in einem Boot« oder »gemeinsam werden wir die Krise meistern« (ausführlich Vobruba), das alles begünstigt konservative Wertvorstellungen und Strategien, die Ausbreitung von Vorurteilen (Ausländerfeindlichkeit) und das Bedürfnis nach "Ruhe und Ordnung«.

Durch die besonderen Umstände der Bundestagswahl 1980 ( $(S t r a u ß-W a h l \ll$ ) wurde der Negativtrend der SPD zwar vorübergehend gestoppt. Die erheblichen Mitgliederverluste seit 1978 (Tabelle 1), die rasche Ausbreitung der Ökologie- und Alternativbewegung und, nach der Wahl, der Friedensbewegung, die Wahlerfolge der Grünen/Alternativen und nicht zuletzt die immer heftigeren innerparteilichen Auseinandersetzungen dokumentierten das Ausmaß, in dem sich die sozialdemokratische Führung von Teilen der eigenen Partei und der Wählerbasis isoliert hatte. Als dann noch die FDP den Absprung aus der Koalition vorbereitete, war es für den Bundeskanzler nur konsequent, die Koalition von sich aus aufzukündigen (17.9.1982).

\subsection{Die Opposition der CDU/CSU}

Der Machtwechsel am 1.10.1982, war nicht das Ergebnis erfolgreicher Oppositionspolitik der Unionsparteien in den Jahren der sozialliberalen Koalition. Die CDU/CSU verfügte keines- 
wegs über eine einheitliche Strategie. Bis zum gescheiterten Mißtrauensvotum gegen Bundeskanzler Brandt (April 1972) und vielleicht sogar bis zur verlorenen Bundestagswahl im November 1972 sah sich die Union eher als »verhinderte Regierungspartei«. Die unerwartete Wahlniederlage führte zu heftigen Auseinandersetzungen um den Oppositionskurs. Während die Linkskonservativen eher einen gemäßigten Reformkurs befürworteten, um verlorengegangene Wähler zurückzugewinnen und die FDP neuerlich für ein Regierungsbündnis zu gewinnen, setzten die Rechtskonservativen auf eine Konfrontationspolitik, deren Ziel letztlich die Vernichtung der FDP war. Die »Südschiene« um Strauß, Filbinger und Dregger wertete das Zusammengehen der FDP mit der SPD im Grunde als unverzeihlichen Klassenverrat. Man hoffte, durch heftiges propagandistisches Trommelfeuer gegen die angeblich sozialistische und den »Ausverkauf Deutschlands « betreibende Koalition die antisozialistischen Ressentiments der FDP-Wähler zu mobilisieren und diese - vor die Alternative »Freiheit oder Sozialismus« gestellt - für die Alleinherrschaft der Union gewinnen zu können.

Die Strategie der Polarisierung zeitigte bei Landeswahlen teilweise beste Ergebnisse. In Bayern erzielte die CSU 1970 erstmalig die absolute Mehrheit (56,4\%) und baute sie 1974 auf $62,1 \%$ aus. Ähnlich in Baden-Württemberg: Hier verbesserte sich die CDU von 44,2\% (1968) über 52,9\% (1972) auf 56,7\% (1976). In Rheinland-Pfalz erreichte die CDU erstmalig 1971 die absolute Mehrheit (50,0\%) und steigerte sich 1975 auf 53,9\%. In Hessen schließlich brachte Alfred Dregger seine Partei 1970 auf 39,7 \% (gegenüber 26,4 \% im Jahre 1966) und 1974 sogar auf 47,3\% (vgl. a. Tabelle 9). Auch die 1969 einsetzenden organisatorischen Anstrengungen zur Umgestaltung der CDU von einer Kanzler-fixierten Honoratiorenpartei zu einer gut organisierten Massenpartei machten rasche Fortschritte. Allein zwischen 1970 und 1977 verdoppelte sich ihre Mitgliederschaft (Tabelle 1). Freilich unterblieb die programmatische Erneuerung. Weder die »Mannheimer Erklärung« von 1975 noch das 1978 verabschiedete Grundsatzprogramm brachten erkennbare Innovationen (ausführlicher U. Schmidt, S. 514 ff.).

Als Programmersatz dienten die politische Polarisierung und, Ende der siebziger, Anfang der achtziger Jahre eine ideologische Großoffensive gegen die Wirtschaftspolitik der SPD. Vor der Bundestagswahl 1980 hatte sich der rechtskonservative Flügel endlich mit seinem Kanzlerkandidaten Strauß durchgesetzt. Wie schon 1972 ging auch jetzt die Polarisierungsstrategie zugunsten der Sozialliberalen aus, die Union verlor, die FDP gewann Stimmen, und die SPD stagnierte (Tabelle 8). Die Regierungskoalition wurde noch einmal durch externe Faktoren gefestigt. In dem durch die Wirtschaftskrise und vor allem durch die Massenarbeitslosigkeit geprägten gesellschaftlichen Klima gelang es der Union jedoch zunehmend erfolgreicher, unter Ausnutzung der wirtschaftspolitischen Differenzen innerhalb der sozialliberalen Koalition, die SPD für die Krise verantwortlich zu machen. Die mit der Reformpolitik verbundenen und schon immer als Sozialismus gebrandmarkten Vergesellschaftungseffekte lieBen sich mit wachsender Resonanz in den Massenmedien, beim Besitzbürgertum, bei den Mittelschichten und selbst bei Teilen der Facharbeiterschaft als Versorgungsstaat und Bürokratismus, als Bevormundung und Behinderung der Wirtschaft, als $»$ Mißwirtschaft« geißeln. Mit den monoton wiederholten Parolen $»$ wir haben jahrelang über unsere Verhältnisse gelebt « und »Leistung muß sich wieder lohnen" gelang es der CDU/CSU, die Zustimmung in der Bevölkerung für die neoliberale Krisenstrategie zu vergrößern. Auf den wirtschaftspolitischen Rezepten Ludwig Erhards, so wurde suggeriert, basierte das "Wirtschaftswunder «, damit läßt sich auch die gegenwärtige Talfahrt stoppen, wenn alle "den Gürtel enger schnallen«. 
Tabelle 9: Bundestagswablergebnisse von CDU/CSU, FDP und SPD in den Ländern 1972-1983 (Zweitstimmen i.v.H.)

\begin{tabular}{|c|c|c|c|c|}
\hline Land & Jahr & CDU/CSU & FDP & SPD \\
\hline \multirow[t]{4}{*}{ Schleswig-Holstein } & 1972 & 42,0 & 8,6 & 48,6 \\
\hline & 1976 & 44,1 & 8,8 & 46,4 \\
\hline & 1980 & 38,9 & 12,7 & 46,7 \\
\hline & 1983 & 46,5 & 6,3 & 41,7 \\
\hline \multirow[t]{4}{*}{ Hamburg } & 1972 & 33,3 & 11,2 & 54,4 \\
\hline & 1976 & 35,9 & 10,2 & 52,6 \\
\hline & 1980 & 31,2 & 14,1 & 51,7 \\
\hline & 1983 & 37,6 & 6,3 & 47,4 \\
\hline \multirow[t]{4}{*}{ Niedersachsen } & 1972 & 42,7 & 8,5 & 48,1 \\
\hline & 1976 & 45,7 & 7,9 & 45,7 \\
\hline & 1980 & 39,8 & 11,3 & 46,9 \\
\hline & 1983 & 45,6 & 6,9 & 41,3 \\
\hline \multirow[t]{4}{*}{ Bremen } & 1972 & 29,6 & 11,1 & 58,1 \\
\hline & 1976 & 32,5 & 11,8 & 54,0 \\
\hline & 1980 & 28,8 & 15,1 & 52,5 \\
\hline & 1983 & 34,2 & 6,5 & 48,7 \\
\hline \multirow[t]{4}{*}{ Nordrhein-Westfalen } & 1972 & 41,0 & 7,8 & 50,4 \\
\hline & 1976 & 44,5 & 7,8 & 46,9 \\
\hline & 1980 & 40,6 & 10,9 & 46,8 \\
\hline & 1983 & 45,2 & 6,4 & 42,8 \\
\hline \multirow[t]{4}{*}{ Hessen } & 1972 & 40,3 & 10,2 & 48,5 \\
\hline & 1976 & 44,8 & 8,5 & 45,7 \\
\hline & 1980 & 40,6 & 10,6 & 46,4 \\
\hline & 1983 & 44,3 & 7,6 & 41,6 \\
\hline \multirow[t]{4}{*}{ Rheinland-Pfalz } & 1972 & 45,9 & 8,1 & 44,9 \\
\hline & 1976 & 49,9 & 7,6 & 41,7 \\
\hline & 1980 & 45,6 & 9,8 & 42,8 \\
\hline & 1983 & 49,6 & 7,0 & 38,4 \\
\hline \multirow[t]{4}{*}{ Baden-Württemberg } & 1972 & 49,8 & 10,2 & 38,9 \\
\hline & 1976 & 53,3 & 9,1 & 36,6 \\
\hline & 1980 & 48,5 & 12,0 & 37,2 \\
\hline & 1983 & 52,6 & 9,0 & 31,1 \\
\hline \multirow[t]{4}{*}{ Bayern } & 1972 & 55,1 & 6,1 & 37,8 \\
\hline & 1976 & 60,0 & 6,2 & 32,8 \\
\hline & 1980 & 57,6 & 7,8 & 32,7 \\
\hline & 1983 & 59,5 & 6,2 & 28,9 \\
\hline \multirow[t]{4}{*}{ Saarland } & 1972 & 43,4 & 7,1 & 47,9 \\
\hline & 1976 & 46,2 & 6,6 & 46,1 \\
\hline & 1980 & 42,3 & 7,8 & 48,3 \\
\hline & 1983 & 44,8 & 6,0 & 43,8 \\
\hline
\end{tabular}

Quelle: Angaben des Statistischen Bundesamtes. 
Dem Druck dieser Propagandaoffensive unterlag vor allem die FDP, deren rechter, "wirtschaftsliberaler "Flügel um Friderichs und Lambsdorff schon seit Mitte der siebziger Jahre mit der Union in wirtschaftspolitischen Fragen mehr übereinstimmte als mit der SPD. Am 1. Oktober 1982 ermöglichte die Mehrheit der FDP-Bundestagsfraktion die Wahl Helmut Kohls zum Bundeskanzler. Die CDU/CSU hatte sich unter den veränderten gesellschaftlichen Verhältnissen zu einer politischen Integrationskraft entwickeln können, die die Wiedererlangung von Stabilität zu leisten versprach.

\subsection{Die Wende der FDP}

Die FDP steht traditionell als Juniorpartner jeder Koalitionsregierung vor dem Problem, die Notwendigkeit ihrer Existenz durch ein eigenes politisches Profil (»liberales Korrektiv«) unter Beweis zu stellen. In dem Maße wie die Konfrontationspolitik der CDU/CSU den Liberalen das Image eines Anhängsels der SPD oder gar eines Steigbügelhalters des Sozialismus anzuheften drohte, mehrte sich innerhalb der FDP das Bedürfnis nach verstärkter Eigenständigkeit. Daß sie diese in der Wirtschafts- und Sozialpolitik und in der Mitbestimmungsfrage suchte, war nicht nur durch die sich seit Mitte der siebziger Jahre verschärfende Wirtschaftskrise verursacht. Damit konnte sie zugleich auch die Angriffe der Union parieren und sich als Sachwalter des Wirtschaftsliberalismus in der Tradition Ludwig Erhards darstellen. Die 1977 verabschiedeten und nicht unwesentlich von Graf Lambsdorff beeinflußten "Kieler Thesen" zielten auf eine streng marktwirtschaftlich orientierte Krisenlösung und wurden weithin als Affront gegen den Koalitionspartner und als Hinwendung zur CDU/ CSU interpretiert. Bereits 1975 hatten sich die Liberalen bei der Wahl in Rheinland-Pfalz auf eine Koalitionsaussage zugunsten der CDU festgelegt (die damals allerdings eine absolute Mehrheit erreichte), 1977 trat die FDP im Saarland und in Niedersachsen in CDU-geführte Landesregierungen ein, 1981 ermöglichte die Berliner FDP die Wahl Richard v. Weizsäckers zum Regierenden Bürgermeister, und 1982 schließlich machte die hessische FDP bei den Landtagswahlen eine Koalitionsaussage zugunsten der CDU.

Da sich die FDP gleichzeitig verbal vehement zur Bonner Koalition bekannte, trug diese Zick-Zack-Politik eher zur Verunsicherung von Mitgliedern und Wählern bei. Ihre Einbrüche bei Landeswahlen (s. Tabelle 10) - die FDP ist gegenwärtig nur noch in vier Landtagen vertreten- waren aber auch durch die Erfolge der Grünen/Alternativen bedingt, mit denen sie um den »eher kritischen, mobilen und aufgeschlossenen Wähler «(Verbeugen, S. 79) konkurrierte. Wenig aussichtsreich allerdings, da sich die FDP-Bundesminister 1977/78 weigerten, Parteibeschlüsse in Bezug auf ein Moratorium für die Kernenergie auszuführen. Galt der Umweltschutz noch Anfang der siebziger Jahre als ein wichtiges FDP-Anliegen, so verärgerte die Partei nun eine potentielle Wählerschaft. Unter dem starken Einfluß der 1978 entstandenen rechtsgerichteten »Liberalen Gesellschaft« und des 1980 in der Bundestagsfraktion gebildeten wirtschaftsliberalen »Wurbs-Kreises« konzentrierte sich die Partei gerade auch auf Druck ihrer besitzbürgerlichen Anhänger nach der Bundestagswahl 1980 auf die Haushaltspolitik und forderte eine radikale "Sanierung der Staatsfinanzen« zu Lasten vor allem sozial schwacher Schichten, (die auch nicht als FDP-Wähler in Betracht kommen). Im August 1981 veröffentlichte Genscher seinen »Wende-Brief«. Die folgenden Haushaltsberatungen verliefen dann unter heftigen Auseinandersetzungen zwischen FDP und SPD, die, mit Rücksicht auf ihre soziale Basis, den neoliberalen Dogmen der Gruppe um Lambsdorff nicht zustimmen konnte. Dieser hatte am 9. September 1982 ein wirtschaftspolitisches Memorandum 
Tabelle 10: Wablergebnisse der FDP in den Ländern (Zweitstimmen i.v.H.)

\begin{tabular}{lcccccc}
\hline & BTW 76 & LW 76-802 & BTW 80 & LW 80-83 & BTW 83 & LW 1983-4 \\
\hline Schleswig-Holsrein & 8,8 & 5,7 & 12,7 & - & 6,3 & 2,2 \\
Hamburg1 & 10,2 & 4,8 & 14,1 & $4,9 / 2,6$ & 6,3 & \\
Niedersachsen & 7,9 & 4,2 & 11,3 & 5,9 & 6,9 & \\
Bremen & 11,8 & 10,7 & 15,1 & - & 6,5 & 4,6 \\
Nordrhein-Wesfalen & 7,8 & 4,98 & 10,9 & - & 6,4 & \\
Hessen & 8,5 & 6,6 & 10,6 & 3,1 & 7,6 & \\
Rheinland-Pfalz & 7,6 & 6,4 & 9,3 & 3,5 & 7,0 & \\
Baden-Württemberg & 9,1 & 8,3 & 12,0 & - & 9,0 & 7,2 \\
Bayern & 6,2 & 6,2 & 7,8 & 3,5 & 6,2 & \\
Saarland & 6,6 & 6,9 & 7,8 & - & 6,0 & \\
Berlin(West) & - & 8,1 & - & 5,6 & - & \\
\hline
\end{tabular}

11982 zwei Landeswahlen: 6.6. und 19.12.

2 Landeswahlen zwischen 1976 und 1980.

3 Landeswahlen zwischen 1980 und 1983.

4 Landeswahlen nach 1983.

Quelle: Angaben des Statistischen Bundesamtes.

»Konzept für eine Politik zur Überwindung der Wachstumsschwäche und zur Bekämpfung der Arbeitslosigkeit« vorgelegt, das der Bundeskanzler als »Scheidungsbrief« auffaßte und wohl auch auffassen sollte. Am 17. September kündigte Helmut Schmidt jedenfalls die Koalition auf, und am 1. Oktober 1982 unterstützte die FDP das von der CDU/CSU eingebrachte konstruktive Mißtrauensvotum im Bundestag.

Die Wende der FDP vollzog sich mithin in zwei Schritten: Zwischen den Bundestagswahlen 1976 und 1980 eröffnete sie sich die Möglichkeit einer Option zugunsten der Union, um dem Negativ-Sog der SPD zu entraten. Nach der Bundestagswahl 1980 sah sie sich um des Überlebens willen zu dem - taktisch schlitzohrigen und mit Blick auf ihre eindeutige Wahlaussage bei der Bundestagswahl 1980 auch den Tatbestand der Wählertäuschung erfüllenden Schritt des Koalitionswechsels veranlaßt, der nach der Logik der Lage der Liberalen jedoch auf der Tagesordnung stand. Schließlich war die Existenz der FDP als liberales Korrektiv in einem Drei-Parteien-System aufs höchste gefährdet. Die FDP war von den Grünen/Alternativen zur nvierten« Partei degradiert worden und drohte schlicht überflüssig zu werden, wenn es ihr nicht gelingen würde, auch zum Preis der Abspaltung eines Teils der Linksliberalen und auf die Gefahr weiterer Niederlagen bei Landeswahlen hin, sich wieder für den Prozeß der öffentlichen Herrschaft unentbehrlich zu machen. Dies ist ihr zunächst gelungen.

\subsection{Die Grünen/Alternativen}

Stellen die Grünen/Alternativen ihrem Wesen nach auch eine Anti-Massenlegitimationspartei dar, so verdanken sie ibre unmitrelbare Entstehung doch nicht dem Bedürfnis, eine parteipolitische Alternative zu den staatstragenden Parteien zu bilden. Vielmehr befand sich die Anti-Atom-Bewegung 1977 in einer Sackgasse (ausführlich: Klotzsch/Stöss, S. 1513 ff.): 
Der außerparlamentarische Kampf hatte zu teilweise bürgerkriegsähnlichen Auseinandersetzungen (Brokdorf, Grohnde) geführt, bei denen Polizei und Bundesgrenzschutz Sieger bleiben mußten und die gerade nicht erhoffte Wirkung hatten, daß innerhalb der etablierten Parteien ein Umdenken in Fragen der Wachstums-, Umwelt- und Atompolitik einsetzte. Die Initiatoren der frühen grünen Parteibildungen entstammten zumeist alteingesessenen Umweltschutzverbänden, den etablierten Parteien oder weithin unbekannten Kleinparteien, die den Barrikadenkampf sowieso ablehnten, radikale Elemente isolieren wollten und im Interesse der Sache eher traditionelle Formen politischer Einflußnahme bevorzugten. Engagiert waren aber auch Reste der gescheiterten »K-Gruppen« oder maoistischer Parteien, die in der Ökologiebewegung eine Massenbasis witterten. Tabelle 11 dokumentiert die Heterogenität der »Wahlbewegung«, die mit der Gründung der Bundespartei »Die Grünen« (Januar 1980) nur teilweise beseitigt werden konnte. Erst die Abspaltung des konservativen Flügels nach der Bundestagswahl 1980 und die Gründung der - bei Wahlen erfolglosen - ÖkologischDemokratischen Partei (ÖDP) um Herbert Gruhl im Oktober 1981 ermöglichten eine Konsolidierung der Bundespartei.

Thr rasches Mitgliederwachstum(Tabelle 1) dürfte vor allem deswegen möglich gewesen sein, weil die Ökologiebewegung als Rekrutierungsfeld zur Verfügung stand. Diese scheint den Zenit ihrer Entwicklung jedoch überschritten zu haben, zumal die Wirtschaftskrise und die sich rapide verschlechternde Finanzlage der Gemeinden den Spielraum für ökologieschädliche Großprojekte einschränkt. Im Zusammenhang mit den Auseinandersetzungen um den "Nato-Doppelbeschluß « entwickelten sich die Grünen/Alternativen dann auch zu einer der Speerspitzen der Friedensbewegung, die mit Sicherheit ein weiteres Rekrutierungsfeld für die neuen Parteien darstellt.

Die Wählerschaft der Grünen/Alternativen setzt sich zum Teil aus Erstwählern, zum Teil aber auch aus ehemaligen Anhängern der sozialliberalen Koalition zusammen, die von deren ursprünglicher Reformpathos begeistert, von der Politik des technokratischen Krisenmanagements dann aber tief enttäuscht waren. Rein zahlenmäßig ist davon zuallererst die FDP betroffen, die bereits 1978 beim Auftreten der Bunten Liste in Hamburg und der GLU in Niedersachsen die Fünfprozenthürde nicht nehmen konnte.

Die Wahlerfolge der Grünen/Alternativen lassen sich mit sozialstrukturellen Faktoren nicht hinreichend erklären. Thre Wählerbasis (über die Mitglieder liegen keinerlei Angaben vor) rekrutiert sich aus allen sozialen Schichten, überproportional vertreten sind die unteren Altersgruppen und die Mittelschichten mit mittleren und höheren Bildungsabschlüssen(s.a. Tabelle 12). Erfolgreich sind sie vor allem in urbanen Milieus und im regionalen Einzugsbereich von hochpolitisierten ökologischen Konflikten. Gemeinsam ist den grün-alternativen Wählern vor allem eine "post-materialistische« Orientierung (ein Phänomen, das jedoch weit in die Basis der Massenlegitimationsparteien hineinreicht) und das Bedürfnis, mehr Einfluß auf die politischen Entscheidungen zu erringen. Dies hat ihnen auch in der sozialwissenschaftlichen Theorie den Ruf einer gesellschaftspolitischen »Gegenelite« in der außerparlamentarischen »Gegenwelt« (Feist/Liepelt 1983, S. 89 ff.) eingebracht, deren Entstehung zwar durch die von der sozialliberalen Koalition betriebenen Bildungsexpansion und durch den ihr zu verdankenden Ausbau des Wohlfahrtsstaates gefördert worden sei, die bei den gegenwärtig restriktiven Arbeitsmarktbedingungen aber kaum Zukunftschancen besäßen. Diese zu verbessern sei Ziel der, auf den Ausbau des nicht-produktiven Sektors der Wirtschaft gerichteten, Politik der Grünen/Alternativen (Bürklin, M.G. Schmidt 1984).

Tatsächlich wird man Existenz und Erfolg der Grünen/Alternativen in binnenstruktureller 
Tabelle 11: Wablergebnisse der Grünen/Alternativen (Zweitstimmen i.v.H.)

\begin{tabular}{|c|c|c|c|c|}
\hline \multicolumn{2}{|l|}{ Wahl } & Parte $/$ Liste & ZwSt i.v.H. & Mandate \\
\hline \multicolumn{5}{|c|}{ Europawahlen } \\
\hline & 1979 & Die Grünen & 3,2 & 0 \\
\hline & 1984 & Die Grïnen & 8,2 & 7 \\
\hline \multicolumn{5}{|c|}{ Bundestagswahlen } \\
\hline & 1980 & Die Grünen & 1,5 & 0 \\
\hline \multirow{2}{*}{\multicolumn{2}{|c|}{1983}} & Die Grünen & 5,6 & 27 \\
\hline & & ÖDP & 0,0 & 0 \\
\hline \multicolumn{5}{|c|}{ Landeswahlen } \\
\hline \multicolumn{5}{|c|}{ Schleswig-Holstein } \\
\hline LW & 1979 & GLSH & 2,4 & 0 \\
\hline EW & 1979 & Die Grünen & 2,7 & 0 \\
\hline BTW & 1983 & Die Grünen & 1,4 & 1 \\
\hline \multirow[t]{2}{*}{$\mathrm{LW}$} & 1983 & Die Grünen & 3,6 & 0 \\
\hline & & DGL & 0,1 & 0 \\
\hline \multirow[t]{2}{*}{ EW } & 1984 & Die Grünen & 8,2 & - \\
\hline & & ÖDP & 0,2 & 0 \\
\hline \multicolumn{5}{|c|}{ Hamburg } \\
\hline \multirow[t]{2}{*}{ LW } & 1978 & BLW & 3,5 & 0 \\
\hline & & GLU & 1,0 & 0 \\
\hline EW & 1979 & Die Grünen & 3,5 & 0 \\
\hline BTW & 1980 & Die Grüinen & 2,3 & 0 \\
\hline \multirow[t]{2}{*}{ LW } & $1982(1)$ & GAL & 7,7 & 9 \\
\hline & & ÖDP & 0,2 & 0 \\
\hline LW & $1982(2)$ & GAL & 6,8 & 8 \\
\hline BTW & 1983 & Die Grünen & 8,2 & 1 \\
\hline \multirow[t]{2}{*}{ EW } & 1984 & Die Grünen & 12,7 & - \\
\hline & & ÖDP & 0,2 & 0 \\
\hline \multicolumn{5}{|c|}{ Niedersachsen } \\
\hline LW & 1978 & GLU & 3,9 & 0 \\
\hline EW & 1979 & Die Grünen & 3,6 & 0 \\
\hline BTW & 1980 & Die Grünen & 1,6 & 0 \\
\hline LW & 1982 & Die Grünen & 6,5 & 11 \\
\hline BTW & 1983 & Die Grünen & 5,7 & 4 \\
\hline \multirow[t]{2}{*}{$\mathrm{EW}$} & 1984 & Die Grünen & 8,2 & - \\
\hline & & ÖDP & 0,3 & 0 \\
\hline \multicolumn{5}{|c|}{ Bremen } \\
\hline EW & 1979 & Die Grüinen & 4,7 & 0 \\
\hline \multirow[t]{2}{*}{ LW } & 1979 & BGL & 5,1 & 4 \\
\hline & & $\mathrm{AL}$ & 1,4 & 0 \\
\hline BTW & 1980 & Die Grünen & 2,7 & 0 \\
\hline B'TW & 1983 & Die Grünen & 9,7 & 0 \\
\hline \multirow[t]{3}{*}{ LW } & 1983 & Die Grünen & 5,4 & 4 \\
\hline & & BGL & 2,4 & 0 \\
\hline & & $\mathrm{BAL}$ & 1,4 & 0 \\
\hline \multirow[t]{2}{*}{ EW } & 1984 & Die Grüinen & 11,9 & - \\
\hline & & ÖDP & 0,2 & 0 \\
\hline \multicolumn{5}{|c|}{ Nordrhein-Westfalen } \\
\hline EW & 1979 & Die Grünen & 3,0 & 0 \\
\hline \multirow[t]{3}{*}{ LW } & 1980 & Die Grünen & 3,0 & 0 \\
\hline & & GPD & 0,0 & 0 \\
\hline & & UNU & 0,0 & 0 \\
\hline
\end{tabular}




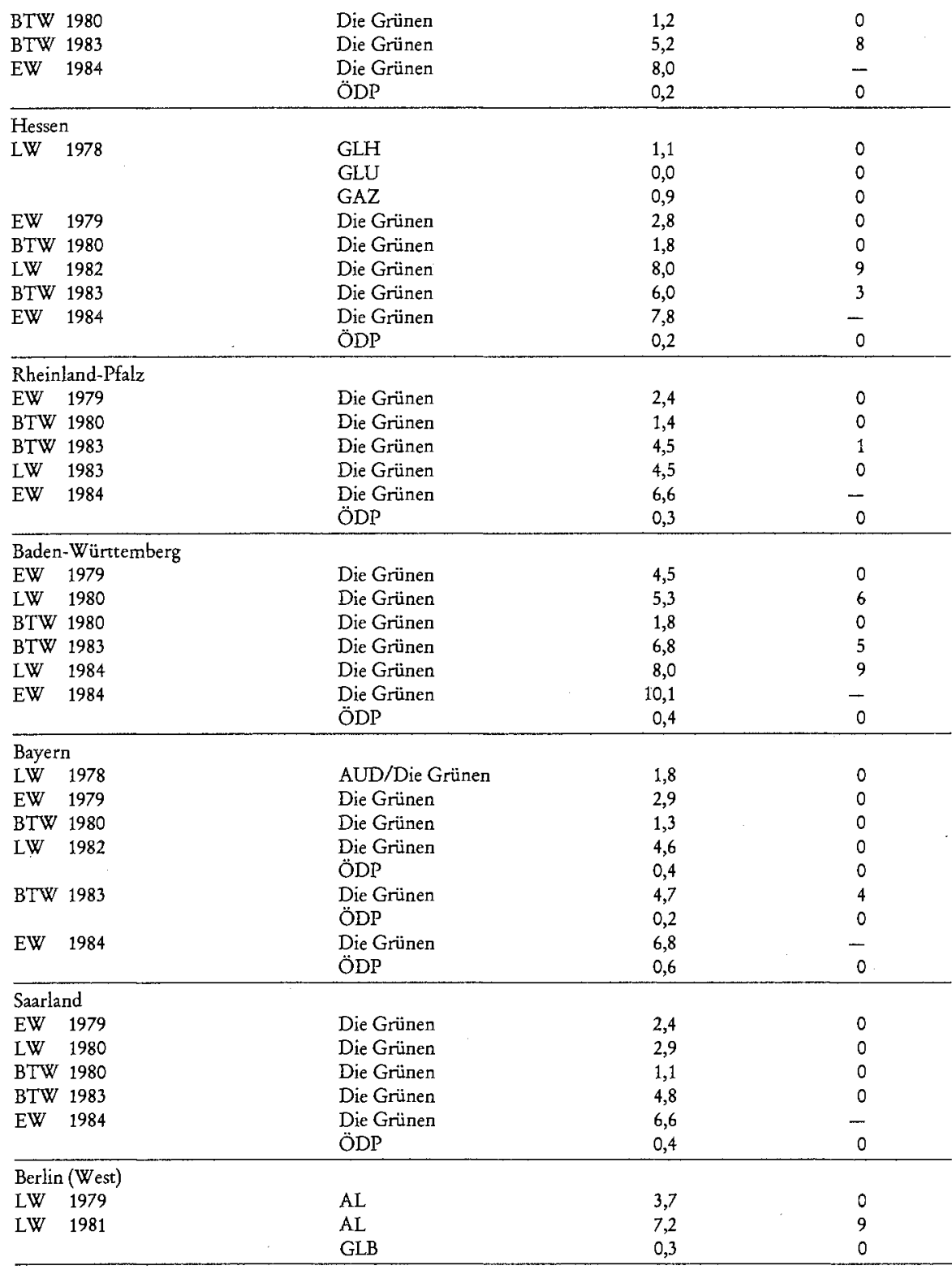

Quelle: Angaben des Statistischen Bundesamtes.

BTW = Bundestagswahlen

$\mathrm{EW}=$ Europawahlen

LW = Landeswahlen 
wie in programmatischer Hinsicht in erster Linie aus der Unfähigkeit der Massenlegitimationsparteien zur flexiblen Reaktion auf neue Problemlagen und geänderte Bedürfnisse der Massen erklären müssen. Deren weitere Entwicklung hängt folglich von der Lernfähigkeit der etablierten Parteien, insbesondere wohl der SPD ab.

\section{Die Ergebnisse der Bundestagswahlen 1980 und 1983}

Die Zweitstimmenergebnisse der vier letzten Bundestagswahlen sind in Tabelle 8 aufgeführt. Die Wahlen 1972 (s. Prokla 26) und 1980 (Anti-Strauß-Wahl) waren stark durch politische Rahmenbedingungen bestimmt, die die Unionsparteien benachteiligten, SPD und FDP dagegen begünstigten. Daß die Grünen nicht schon 1980 die Fünfprozenthürde überwinden konnten, lag aller Wahrscheinlichkeit nach ebenfalls an der Schmidt-Strauß-Polarisierung. Da ihre Anhängerschaft überdurchschnittlich qualifiziert, politisch interessiert und mobil ist und überdies der SPD politisch nahesteht, dürfte sie zu einem großen Teil Schmidt gewählt haben, um einen Kanzler Strauß zu verhindern (Berger/Gibowski/Rotb/Schulte 1983a, S. 50). So konnte die SPD $1980 \mathrm{ihr}$ Ergebnis von 1976 halten, war aber mit knapp $43 \%$ überbewertet. Ähnlich wird der hohe Stimmenanteil der FDP $(10,6 \%)$ zu beurteilen sein. Bei der Bundestagswahl 1983 erübrigte sich für die Angänger der Grünen eine Stimmabgabe nach taktischen Gesichtspunkten, und so zog erstmalig seit 1957 wieder eine "vierte Partei in den Bundestag ein. (Damals hatte es die DP allerdings nicht durch die Überwindung der Sperrklausel, sondern auf dem Wege des »Huckepackverfahrens« geschafft). Die SPD verschlechterte sich auf 38,2 \% (Erststimmenanteil 40,4\%), der FDP gelang mit $7 \%$ wider Erwarten doch der Einzug in das Bundesparlament, und die CDU/CSU wurde mit 48,8 \% (Erststimmenanteil 52,2 \%) deutlich stärkste Partei. Berücksichtigt man, daß $70 \%$ der FDP-Wähler vom Stimmensplitting Gebrauch gemacht und daß davon sechs Zehntel der CDU/CSU und nur ein Zehntel der SPD ihre Erststimme gegeben haben (Berger/Gibowski/Roth/Schulte 1983b, S. 558), dann dürften der FDP auch »Leihstimmen « der CDU zugute gekommen sein. Kaltefleiter (S. 3) schätzt das »wirkliche Potential der CDU/CSU « auf etwa 53 \%. Mag dies auch etwas überzogen sein: Die Unionsparteien hatten 1983 jedenfalls eine realistische Chance, wie 1957 die absolute Mehrheit zu erreichen.

Vergleicht man die tatsächlichen Zweitstimmenergebnisse von 1983 nicht (wegen der besonderen Situation) mit denen von 1980 sondern mit den Resultaten von 1976, dann ergeben sich keine spektakulären Veränderungen. Detailanalysen außer acht gelassen, kann auf Grundlage der ausgezählten Wahlscheine festgestellt werden, daß sich CDU/CSU und FDP unverändert gehalten haben und daß die SPD verloren hat, was die Grünen hinzugewonnen haben. Das Wählerpotential »links von der Mitte liegt also seit 1969 relativ konstant um $43 \%$ (Berger/Gibowski/Roth/Schulte 1983b, S. 576).

Spektakuläre Veränderungen sieht dagegen Kaltefleiter, der den "Wahltriumph « seiner Partei euphorisch in die Nähe des vermeintlichen Wahlwunders der CDU/CSU von 1953 (die Unionsparteien verbesserten sich damals von $31,0 \%$ auf $45,2 \%$; zu den keineswegs verwunderlichen Ursachen s. Prokla 25, S. 22 ff.) rückt, und auch Feist/Krieger/Uttitz (S. 415) sprechen von »ungewöhnlich großen Wählerverschiebungen« seit der Bundestagswahl 1976. Unbestritten ist, daß im Bereich der Wechselwähler, deren Wahlentscheidung von tagespolitischen Einflüssen abhängt, ständig Fluktuation herrscht, und daß gerade diese Gruppe den Unionsparteien 1983 die weitaus größere Kompetenz zur Lösung der ökonomischen Fragen 
zubilligte. Selbst SPD-Anhänger trauten hier besonders häufig der Union mehr Erfolge zu, als ihrer eigenen Partei (Berger/Gibowski/Roth/Schulte 1983b, S. 572 f.). Um die Frage allerdings, ob die SPD-Verluste auch zu Lasten ihrer »Stammwählerschaft « erfolgt sind, ob es also der CDU/CSU gelungen ist, in die traditionelle sozialdemokratische Basis einzubrechen, hat sich eine Kontroverse zwischen der Forschungsgruppe Wahlen (Berger u.a.) und dem infasInstitut (Feist u.a.) entfacht.

Die Tabellen 12 bis 14 präsentieren Umfrageergebnisse der FG Wahlen und zeigen für den Zeitraum zwischen 1976 und 1983, daß sich die SPD bei den Gewerkschaftsmitgliedern insgesamt nicht verschlechtert, bei den gewerkschaftlich organisierten Arbeitern sogar leicht verbessert hat. Auch bei den gelernten Arbeitern ist ein leichter Anstieg festzustellen. Einbrüche sind dagegen bei den ungelernten Arbeitern und bei den gewerkschaftlich nicht gebundenen Beamten/Angestellten zu konstatieren. Die FGW-Zahlen sprechen also dafür, daß sich die SPD bei ihren "Stammwählern« (gewerkschaftlich organisierte Arbeiter) behaupten konnte und daß ihre Verluste vor allem im Bereich der Mittelschichten anfielen. Das Eis der SPD-Wählerschaft schmilzt, so Berger/Giboreski/Roth/Schulte (1984), zuerst an den Rändern.

Nach den Umfrageergebnissen von infas (Feist/Krieger/Uttitz, Feist/Krieger/Smid) dagegen ist die Bundestagswahlabsicht von 1976 auf 1983 bei ungelernten Arbeitern von $51 \%$ auf $53 \%$ zwar leicht gestiegen, bei Facharbeitern jedoch von $61 \%$ auf $54 \%$ gesunken. Die angelernten Arbeiter stagnieren bei $59 \%$. Der Anteil der gewerkschaftlich organisierten Arbeiter unter den SPD-Anhängern hat sich nach infas wie folgt entwickelt: $197671 \%, 198068 \%$, $198362 \%$. Nun behaupten die FGW-Autoren (Berger/Giboreski/Rotb/Scbulte 1984), daß diese Datenreihen wegen hier nicht zu erörternder Verfahrensmängel die Realität nur verzerrt wiedergeben. Dies klingt plausibel. Es ist jedoch in Rechnung zu stellen, daß Umfragen vor oder nach einer Wahl das Wahlergebnis immer verzerrt abbilden und folglich stets mit Vorsicht zu interpretieren sind. Das Datenmaterial von infas signalisiert die bereits erkennbare und mit Blick auf den politischen Kontext auch plausible Gefahr, daß die SPDVerluste auch den Kern ihrer »Stammwähler» betreffen. Auf die demobilisierenden Folgen sozialdemokratischer Krisenprophylaxe wurde bereits hingewiesen, ebenso auf die Spannungen zwischen SPD und Gewerkschaften. So ist es keineswegs verwunderlich, daß die Kernanhänger der Partei inaktiv geworden sind, nicht zur Wahlurne gehen oder sogar die $\mathrm{CDU} / \mathrm{CSU}$ wählen.

Im Ergebnis haben auch die letzten beiden Bundestagswahlen die enorme Stabilität und Integrationsfähigkeit des westdeutschen Parteiensystems unter Beweis gestellt. Der Erfolg der Grünen/Alternativen widerspricht diesem Befund nicht, er dokumentiert eher die Flexibilität, mit der das politische System auf Legitimationsdefizite und Desintegrationstendenzen zu reagieren in der Lage ist. Auch die ökonomische Krise hat bislang nicht zu einer den Basiskonsens bedrohenden Verschärfung der Verteilungskämpfe geführt. Nicht einmal der Koalitionswechsel vom Herbst 1982 ist ausschließlich ihr Verdienst. Die Massenarbeitslosigkeit schlägt sich ebenfalls nicht in spektakulär veränderten Parteipräferenzen nieder. Nach einer Unifrage vom Februar 1983 (Kieselbach, S. 811) bevorzugen $53 \%$ der Arbeitslosen die SPD, $31,6 \%$ die CDU/CSU, 11,1 \% die Grünen und 1,7\% die FDP. Bislang hat also das politische System der Bundesrepublik die kapitalistische Krise erfolgreich verarbeitet. Die kapitalismusfunktionalen Anpassungsleistungen der Politik waren vor allem deshalb möglich, weil das Konsenspotential in der Gesellschaft immer noch enorm groß ist.

Dies muß nicht notwendigerweise so bleiben. Zwar wird sich die regierende Bürgerkoalition 
Wablentscheidung und Beruf, Gewerkschaftsmitgliedschaft und Konfession

Tabelle 12: Wablentscheidung und Beruf des Haushaltsvorstands (i.v.H.)

\begin{tabular}{|c|c|c|c|c|c|c|c|c|c|c|c|c|c|c|c|c|c|c|}
\hline \multirow[b]{3}{*}{ Wahlentscheidung } & \multirow{2}{*}{\multicolumn{3}{|c|}{ Alle Befragte }} & \multicolumn{6}{|c|}{ Arbeiter } & \multicolumn{5}{|c|}{ Angestellte und Beamte } & \multirow{2}{*}{\multicolumn{4}{|c|}{$\begin{array}{l}\text { Selbständige } \\
\text { bis mittlere }\end{array}$}} \\
\hline & & & & \multicolumn{3}{|c|}{ ungelernt } & \multicolumn{3}{|c|}{ gelernt } & \multicolumn{3}{|c|}{ bis mittlere } & \multicolumn{2}{|c|}{ leitende } & & & & \\
\hline & 1976 & 1980 & 1983 & 1976 & 1980 & 1983 & 1976 & 1980 & 1983 & 1976 & 1980 & 1983 & 1976 & 1980 & 1983 & 1976 & 1980 & 1983 \\
\hline CDU/CSU & 48 & 43 & 51 & 36 & 39 & 45 & 44 & 32 & 43 & 45 & 41 & 56 & 58 & 52 & 56 & 63 & 56 & 61 \\
\hline FDP & 8 & 12 & 5 & 2 & 6 & 3 & 6 & 11 & 2 & 10 & 15 & 4 & 14 & 10 & 3 & 5 & 13 & 11 \\
\hline SPD & 43 & 44 & 38 & 62 & 55 & 50 & 49 & 57 & 52 & 44 & 42 & 34 & 28 & 37 & 24 & 32 & 28 & 26 \\
\hline Grüne & - & 1 & 6 & - & 0 & 1 & - & 0 & 3 & - & 2 & 7 & - & 1 & 17 & - & 0 & 2 \\
\hline$n=$ & 1114 & 968 & 931 & 109 & 87 & 84 & 342 & 257 & 293 & 365 & 342 & 306 & 89 & 80 & 61 & 123 & 99 & 80 \\
\hline
\end{tabular}

Tabelle 13: Wablentscheidung und Gewerkschaftsmitgliedschaft nach Berufgruppen (i.v.H.)

\begin{tabular}{|c|c|c|c|c|c|c|c|c|c|c|c|c|c|c|c|c|c|c|}
\hline \multirow[b]{3}{*}{ Wahlentscheidung } & \multicolumn{6}{|c|}{ Alle Befragte } & \multicolumn{6}{|c|}{ Arbeiter } & \multicolumn{6}{|c|}{ Angestellte und Beamte } \\
\hline & \multicolumn{3}{|c|}{ Mitglieder } & \multicolumn{3}{|c|}{ Nichtmitglieder } & \multicolumn{3}{|c|}{ Mitglieder } & \multicolumn{3}{|c|}{ Nichtmitglieder } & \multicolumn{3}{|c|}{ Mitglieder } & \multicolumn{3}{|c|}{ Nichtmitglieder } \\
\hline & 1976 & 1980 & 1983 & 1976 & 1980 & 1983 & 1976 & 1980 & 1983 & 1976 & 1980 & 1983 & 1976 & 1980 & 1983 & 1976 & 1980 & 1983 \\
\hline $\mathrm{CDU} / \mathrm{CSU}$ & 35 & 29 & 36 & 55 & 48 & 56 & 35 & 29 & 34 & 48 & 36 & 51 & 35 & 29 & 41 & 55 & 47 & 61 \\
\hline FDP & 9 & 10 & 3 & 8 & 13 & 5 & 6 & 8 & 3 & 5 & 11 & 2 & 12 & 14 & 4 & 10 & 13 & 4 \\
\hline SPD & 56 & 58 & 56 & 36 & 38 & 31 & 58 & 62 & 64 & 47 & 53 & 42 & 52 & 55 & 48 & 34 & 37 & 26 \\
\hline Grüne & - & 3 & 5 & - & 1 & 7 & - & 1 & 0 & - & 0 & 5 & - & 2 & 7 & - & 3 & 9 \\
\hline $\mathbf{n}=$ & 392 & 271 & 278 & 700 & 675 & 639 & 204 & 153 & 163 & 241 & 181 & 205 & 161 & 100 & 99 & 282 & 312 & 264 \\
\hline
\end{tabular}




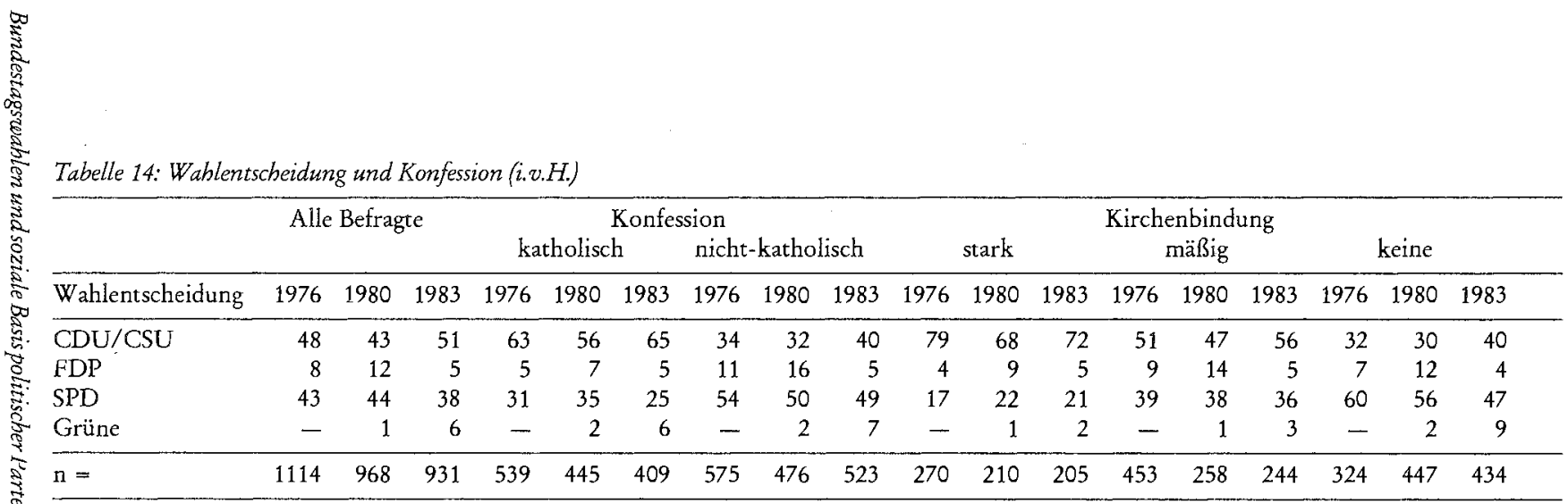

Quelle: Berger/Gibowski/Roth/Schulte 1983b, S.578, 580.

Tabelle 8: Bundestagswahlergebnisse 1972.1983 Zweitstimmen (i.v.H.)

\begin{tabular}{|c|c|c|c|c|c|c|c|c|c|c|c|c|}
\hline & \multicolumn{2}{|c|}{$\mathrm{CDU} / \mathrm{CSU}$} & \multicolumn{2}{|l|}{ FDP } & \multicolumn{2}{|l|}{ SPD } & \multicolumn{2}{|l|}{ DKP } & \multicolumn{2}{|l|}{ NPD } & \multicolumn{2}{|c|}{ Die Grünen } \\
\hline & ZwSt.i.v.H. & Mand. & ZwSt.i.v.H. & Mand. & ZwSt.i.v.H. & Mand. & ZwSt.i.v.H. & Mand. & $Z_{w S t . i . v . H .}$ & Mand. & ZwSt.i.v.H. & Mand. \\
\hline 1972 & 44,9 & 225 & 8,4 & 41 & 45,8 & 230 & 0,3 & 0 & 0,6 & 0 & - & - \\
\hline 1976 & 48,6 & 243 & 7,9 & 39 & 42,6 & 214 & 0,3 & 0 & 0,3 & 0 & - & 一 \\
\hline 1980 & 44,5 & 226 & 10,6 & 53 & 42,9 & 218 & 0,2 & 0 & 0,2 & 0 & 1,5 & 0 \\
\hline 1983 & 48,8 & 244 & 7,0 & 34 & 38,2 & 193 & 0,2 & 0 & 0,2 & 0 & 5,6 & 27 \\
\hline
\end{tabular}

\pm Quelle: Angaben des Statistischen Bundesamtes. 
larum bemühen, die Demontage des Sozialstaats bis auf seine Grundfesten zu vermeiden und den Verlust von Sozialstaatlichkeit durch die vermehrte Produktion von Gemeinschaftsideologien zu kompensieren. Da aber die Konsensfähigkeit einer Gesellschaft von subjektiv akzeptablen Lebens-, Arbeits- und Verwertungsbedingungen abhängt, wird sich - infolge andauernder Massenarbeitslosigkeit, wachsender Armut, verschärfter Konkurrenzkämpfe und rasanter Kapitalvernichtung - die Intensivierung der Verteilungskämpfe (auch ideologisch) nicht verhindern lassen. In dieser Situation sind Sozialdemokratie und Gewerkschaften nachgerade gezwungen, die Interessen der Lohnabhängigen konsequent zu vertreten und mithin dieökonomisch-sozialen Konflikte auch auf der politischen Ebene auszutragen, oder ihre Basis bröckelt ab. Absehbar ist also, daß die Parteien verstärkt die Funktion der Interessenrepräsentation wahrnehmen und so ihren Charakter als Massenlegitimationsparteien allmählich ablegen werden. Selbst wenn im Laufe dieser Entwicklung der Basiskonsens geschwächt wird, ist eine Bedrohung des Verfassungskonsenses höchst unwahrscheinlich.

\section{Literaturverzeichnis}

Johannes Agnoli, Die Transformation der Demokratie, in: Ders./Peter Brückner, Die Transformation der Demokratie, Berlin 1967

Manfred Berger/Wolfgang G. Giboweski/Dieter Roth/Wolfgang Schulte, Bundestagswahl 1976: Politik und Sozialstruktur, in: ZParl, 8. Jg. (1977), H. 2, S. $197 \mathrm{ff}$.

Manfred Berger/Wolfgang G. Giboreski/Dieter Roth/Wofgang Schulte, Stabilität und Wechsel. Eine Analyse der Bundestagswahl 1980, in: Max Kaase/Hans-Dieter Klingemann (Hrsg.), Wahlen und politisches System. Analysen aus Anlaß der Bundestagswahl 1980, Opladen 1983, S. 22 ff. (a)

Manfred Berger/Wofgang G. Giboweski/Dieter Roth/Wolfgang Schulte, Regierungswechsel und politische Einstellungen. Eine Analyse der Bundestagswahl 1983, in: ZParl, 14. Jg. (1983), H. 4, S. 556 ff. (b)

Manfred Berger/Wolfgang G Giboreski/Dieter Roth/Wolfgang Schulte, Das Eis schmilzt zuerst an den Rändern ... Zur Infas-These von den Stammwählerverlusten der SPD, in: ZParl, 15. Jg.(1984), H. 2, S. $305 \mathrm{ff}$.

Bernhard Blanke, Formen und Formwandel des politischen Systems in der bürgerlichen Gesellschaft, in: Staat - Politische Ökonomie: Handbuch 5, Frankfurt a.M./Köln 1977, S. 121 ff.

Bernhard Blanke/Ulrich Jürgens/Hans Kastendiek, Kritik der Politischen Wissenschaft. Analysen von Politik und Ökonomie in der bürgerlichen Gesellschaft, Frankfurt a.M. 1975

Wilhelm P. Bürklin, Ansatzpunkte einer sozialstrukturellen Verankerung der neuen sozialen Bewegungen, in: Politische Willensbildung und Interessenvermittlung. Verhandlungen der Fachtagung der DVPW vom 11.-13. Oktober 1983 in Mannheim, Opladen 1984, S. 566 ff.

Ursula Feist/Hubert Krieger/Menno Smid, Das »kritische« Potential bei der Bundestagswahl 1983: Die bewußte Arbeitnehmerschaft. Eine Antwort auf die Forschungsgruppe Wahlen, in: ZParl, 15. Jg. (1984), H. 1, S. 124 ff.

Ursula Feist/Hubert Krieger/Pavel Vttitz, Das Wahlverhalten der Arbeiter bei der Bundestagswahl 1983, in: Gewerkschaftliche Monatshefte, 34. Jg. (1983), H. 7, S. 414 ff.

Ursula Feist/Klaus Liepelt, Machtwechsel in Raten: Das Parteiensystem auf dem Weg zur Mitte, in: Wahlforschung: Sonden im politischen Markt, Transfer 2, Opladen 1976, S. 26 ff.

Ursula Feist/Klaus Liepelt, Stärkung und Gefährdung der sozialliberalen Koalition. Das Ergebnis der Bundestagswahl vom 5. Oktober 1980, in: ZParl, 12. Jg. (1981), H. 1, S. 34 ff.

Ursula Feist/Klaus Liepeli, Neue Eliten in alten Parteien. Anmerkungen zu einer Nebenfolge der Bildungsreform, in: Max Kaase/Hans-Dieter Klingemann (Hrsg.), Wahlen und politisches System. Analysen aus Anlaß der Bundestagswahl 1980, Opladen 1983, S. 81 ff. 
Forschungsgruppe Wablen e.V., Bundestagswahl 1983, Eine Analyse der Wahl zum 10. Deutschen Bundestag am 6. März 1983, Berichte der FGW Nr. 32, Mannheim, 9. März 1983

Peter Gluchowski/Hans-Joachim Veen, Nivellierungstendenzen in den Wähler- und Mitgliedschaften von CDU/CSU und SPD 1959 bis 1979, in: ZParl, 10. Jg. (1979), H. 3, S. 312 ff.

Michael Th. Greven, Parteien und politische Herrschaft. Zur Interdependenz von innerparteilicher Ordnung und Demokratie in der BRD, Meisenheim a. Glan 1977

Siegfried Heimann, Die Sozialdemokratische Partei Deutschlands, in: Parteien-Handbuch. Die Parteien. der Bundesrepublik Deutschland 1945-1980, hrsg. v. Richard Stöss, Bd. 2, Opladen 1984, S. 2025 ff.

Dietrich Herzog, Politische Karrieren. Selektion und Professionalisierung politischer Führungsgruppen, Opladen 1975

Jürgen Hoffmann, »Das Ende der Fahnenstange« - Sozialdemokratie und keynesianischer Klassenkompromiß in der Bundesrepublik, in: Prokla 49, 12. Jg. (1982), H. 4, S. 9 ff.

Werner Kaltefleiter, Eine kritische Wahl. Anmerkungen zur Bundestagswahl 1983, Aus Politik und Zeitgeschichte. Beilage zur Wochenzeitung Das Parlament, B14/83 v. 9.4.1983, S. $3 \mathrm{ff.}$

Hermann Kaste/Joachim Rascbke, Zur Politik der Volkspartei, in: Wolf-Dieter Narr (Hrsg.), Auf dem Weg zum Einparteienstaat, Opladen 1977, S. 26 ff.

Thomas Kieselbach, Arbeitslosigkeit und politische Orientierungen, in: Gewerkschaftliche Monatshefte, 34. Jg. (1983), H. 12, S. $802 \mathrm{ff}$.

Lilian Klotzsch/Richard Stöss, Die Grünen, in: Parteien-Handbuch. Die Parteien der Bundesrepublik Deutschland 1945-1980, hrsg. v. Richard Stöss, Bd. 2, Opladen 1984, S. 1509 ff.

Wolf-Dieter Narr (Hrsg.), Auf dem Weg zum Einparteienstaat, Opladen 1977

Sigmund Neumann, Die politischen Parteien in Deutschland, Berlin 1932; hier zit. nach der 3. Auflage: Die Parteien der Weimarer Republik, m.e. Einführung von Karl-Dietrich Bracher, Stuttgart usw. 1973

Dieter Oberndörfer, Pespektiven nach dem 3. Oktober 1976. Eine Analyse der Bundestagswahlen, in: Die politische Meinung Nr. 169, 21. Jg. (1976), S. 5 ff.

Theo Schiller, Wird die F.D.P. eine Partei?, in: Wolf-Dieter Narr (Hrsg.), Auf dem Weg zum Einparteienstaat, Opladen 1977, S. $122 \mathrm{ff}$.

Manfred G. Schmidt, Die "Politik der inneren Reformen« in der Bundesrepublik Deutschland 19691976, in: PVS, 19. Jg. (1978), H. 2, S. $201 \mathrm{ff.}$

Manfred G. Schmidt, Der Beitrag der »etablierten Politik«zur Entstehung der neuen sozialen Bewegungen, in: Politische Willensbildung und Interessenvermittlung. Verhandlungen der Fachtagung der DVPW vom 1.1.-13. Oktober 1983 in Mannheim, Opladen 1984, S. $580 \mathrm{ff}$.

Ute Schmidt, Die Christlich Demokratische Union Deutschlands, in: Parteien-Handbuch. Die Parteien der Bundesrepublik Deutschland 1945-1980, hrsg. v. Richard Stöss, Bd. 1, Opladen 1983, S. 490 ff.

Horst W. Schmollinger, Abhängig Beschäftigte in Parteien der Bundesrepublik: Einflußmöglichkeiten von Arbeitern, Angestellten und Beamten, in: Zum Nachdenken '55, hrsg. v.d. Hessischen Landeszentrale für politische Bildung, Wiesbaden 1974 (zunächst: ZParl 1974, S. 58 ff.)

Horst W. Schmollinger/Richard Stöss, Sozialstruktur und Parteiensystem, in: Dietrich Staritz (Hrsg.), Das Parteiensystem der Bundesrepublik. Geschichte - Entstehung - Entwicklung. Eine Einführung, 2. Aufl., Opladen 1980, S. $231 \mathrm{ff.}$

Richard Stöss, Struktur und Entwicklung des Parteiensystems der Bundesrepublik - Eine Theorie, in: Parteien-Handbuch. Die Parteien der Bundesrepublik Deutschland 1945-1980, hrsg. v. Richard Stöss, Bd. 1, Opladen 1983, S. 17 ff.

Hans-Joachim Veen/Peter Gluchowski, Tendenzen der Nivellierung und Polarisierung in den Wählerschaften von CDU/CSU und SPD von 1959 bis 1983 - eine Fortschreibung, in: ZParl, 14. Jg. (1983), H. 4, S. 545 ff.

Günter Verhetgen, Der Ausverkauf. Macht und Verfall der FDP, Reinbek 1984

Georg Vobruba, Gemeinschaftsbewußtsein in der Gesellschaftskrise, in: Ders. (Hrsg.), »Wir siizen alle in einem Boot«. Gemeinschaftsrhethorik in der Krise, Frankfurt a.M./New York 1983, S. 9 ff.

Wabl zum 10. Deutschen Bundestag am 6. März 1983, H. 1: Ergebnisse und Vergleichszahlen früherer 
Bundestags- und Landtagswahlen sowie Strukturdaten für die Bundestagswahlkreise 1983, hrsg. v. Statistischen Bundesamt Wiesbaden, Mainz 1983 (Fachserie 1: Bevölkerung und Erwerbstätigkeit) Wabl zum 10. Deutschen Bundestag am 6. März 1983, H. 3: Endgültige Ergebnisse nach Wahlkreisen, hrsg. v. Statistischen Bundesamt Wiesbaden, Mainz 1983 (Fachserie 1: Bevölkerung und Erwerbstätigkeit) 